\title{
Strong heterogeneity caused by deep mantle layering
}

\author{
Paul J. Tackley \\ Department of Earth and Space Sciences and Institute of Geophysics and Planetary Physics, University of California, 595 \\ Charles Young Drive East, Los Angeles, California 90095, USA (ptackley@ucla.edu)
}

[1] Numerical simulations are used to characterize the volumetric heterogeneity associated with proposed chemical layering in the deep mantle. A globally continuous, undulating layer generates enormous amounts of volumetric heterogeneity, which is caused by lateral variations in the layer boundary depth and by strong hot upwellings arising from the top of the layer, in the middle lower mantle region. In contrast, the heterogeneity in the deepest mantle is very weak. Partial cancellation of the temperature and compositional contributions to seismic velocity could mask the layer itself, but the strong signatures of the thermochemical boundary layer and induced upwellings remain, even when fields are filtered to approximate the resolution of global seismic tomographic models. This distinctive heterogeneity is not observed in global seismic tomographic models. Dense material that is restricted to discontinuous "piles" generates heterogeneity that is more consistent, although not entirely, with seismic observations, with strong heterogeneity at the core-mantle boundary. Radial correlation functions display a characteristic narrowing in the vicinity of any chemical layer but are very sensitive to the applied "seismic tomographic" filtering.

Components: 10,068 words, 11 figures, 1 table.

Keywords: Mantle structure; thermochemical convection; geochemical layering.

Index Terms: 8120 Tectonophysics: Dynamics of lithosphere and mantle — general; 8124 Tectonophysics: Earth's interiorcomposition and state; 1025 Geochemistry: Composition of the mantle.

Received 10 April 2001; Revised 5 October 2001; Accepted 22 December 2001; Published 13 April 2002.

Tackley, P. J., Strong heterogeneity caused by deep mantle layering, Geochem. Geophys. Geosyst., 3(4), 10.1029/ 2001GC000167, 2002.

Theme: Thermochemical Convection in the Earth's Interior

Guest Editors: Peter van Keken, Louis Moresi, and Adrian Lenardic

\section{Introduction}

[2] The thin $\mathrm{D}^{\prime \prime}$ layer at the base of the mantle has commonly been interpreted to be a chemically distinct dense layer [e.g., Davies and Gurnis, 1986; Hansen and Yuen, 1988; Kellogg and King, 1993]. However, it has more recently been proposed that a deep dense layer might extend much further into the lower mantle, either in the form of an undulating but globally continuous $\sim 1000 \mathrm{~km}$ thick layer [Kellogg et al., 1999], or in the form of isolated "piles" of material that may extend up to $\sim 1500 \mathrm{~km}$ into the mantle [Tackley, 1998] and correspond to the seismically imaged "megaplumes" [e.g., Masters et al., 2000; Su and Dziewonski, 1997].

[3] In order to test whether such structures exist in Earth, it is important to establish their observational 
signatures and compare to observations. One possibility already considered is that a seismic reflection occurs from the combined chemical and thermal boundary layer that would exist if the chemical discontinuity is sharp. However, Vidale et al. [2001] modeled and searched for such a middle mantle discontinuity and were unable to find one. If such a layer does exist, it may be that the layer boundary is not sharp and/or that the layer is so uneven that it does not produce a clean reflection.

[4] This paper focuses on the signature of volumetric mantle heterogeneity associated with a dense thermochemical layer, considering both visual patterns and statistical diagnostics such as the radial correlation function (RCF) [Jordan et al., 1993] and spectral heterogeneity map (SHM) [Tackley et al., 1994]. Three three-dimensional (3-D) scenarios are presented: an isochemical reference case and two thermochemical cases, one of which develops isolated "piles" [Tackley, 1998] and one of which develops a continuous layer [Kellogg et al., 1999]. The objective of this study is not to systematically explore parameter space (which is still prohibitively expensive in three dimensions and best done in two dimensions [Hansen and Yuen, 2000; Montague and Kellogg, 2000]) but rather to characterize the observational signature of the proposed structures when they arise in a dynamically self-consistent manner for reasonable mantle parameters. The analysis thus focuses on parameter combinations that lead to thermochemical structures similar to those proposed.

\section{Model}

[5] The physical and numerical models are similar to those by Tackley [1996a, 1998] but with some differences. To summarize, a compressible anelastic approximation is assumed in Cartesian geometry, with thermodynamic properties density and thermal expansivity having a depth-dependence identical to that previously assumed, but thermal conductivity having a profile that is more consistent with recent constraints [Hofmeister, 1999]. Values of nondimensional parameters and their nominal dimensional equivalents are given in Table 1. The parameters are intended to be Earth-like, with the possible exception of viscosity which may be slightly too high. The resulting convective vigor is similar to slightly lower than Earth-like.

[6] The model is heated from within and below, in contrast to the models in [Tackley, 1998], which were heated entirely from below. It has been proposed that hypothesized dense layer contains a higher concentration of heat-producing elements than regular mantle, perhaps accounting for the heat-producing elements that are "missing" from the MORB source [Kellogg et al., 1999]. Thus the internal heating rate is increased by an arbitrary factor of 10 in the dense material compared to regular mantle, and the mantle heating rate is adjusted to give the same total heat input of nondimensional 25 , equivalent to a dimensional value of $65 \mathrm{~mW} / \mathrm{m}^{2}$ (Table 1). No phase changes are included in these initial models for simplicity, though the effect of these on modulating thermochemical convection [e.g., Christensen and Yuen, 1984; van Keken et al., 1996; Weinstein, 1992] certainly deserves further study.

\subsection{Viscosity}

[7] Viscosity is dependent on depth and composition only, with the nondimensional viscosity (nondimensionalized to the viscosity at the surface) given by

$$
\eta(d, C)=[1+29 H(d-0.228)] \exp [d \ln 10-C \ln 100],
$$

where $H$ is the Heaviside step function, $d$ is (nondimensional) depth, and $C$ is composition (varying between 0 for regular mantle and 1 for dense material). The depth-dependence thus consists of a steady (exponential) factor of 10 increases with depth plus a jump of factor 30 at $660 \mathrm{~km}$. It was decided not to include temperature-dependent viscosity in these calculations because it would lead to a rigid lid style of convection that is not representative of Earth [Moresi and Solomatov, 1995; Solomatov, 1995] (although encouraging progress is being made in producing plate tectonic convection in numerical models [Moresi et al., 2000; Moresi and Solomatov, 1998; Tackley, $2000 \mathrm{~d}, 2000 \mathrm{e}])$. Instead, it is noted that the dense material becomes substantially hotter than the overlying mantle and that viscosity in the lower mantle is strongly temperature dependent [Yama- 
Table 1. Thermodynamic Parameters

\begin{tabular}{|c|c|c|c|c|}
\hline Parameter & Symbol & Value & Units & Non- $D$ value \\
\hline \multicolumn{5}{|c|}{ Nondimensional Parameters } \\
\hline T-based surface Rayleigh number & $\mathrm{Ra}_{s}$ & & & $10^{8}$ \\
\hline Total internal heating rate & $\mathrm{H}$ & & & 25 \\
\hline Mean dissipation number & Di & & & 0.441 \\
\hline \multicolumn{5}{|c|}{ Nominal Dimensional Parameters } \\
\hline Temperature scale & $\Delta T$ & 2500 & $\mathrm{~K}$ & 1.0 \\
\hline Density: surface & $\rho_{s}$ & 4000 & $\mathrm{~kg} \mathrm{~m}^{-3}$ & 1.0 \\
\hline Density: CMB & $\rho_{c}$ & 5600 & $\mathrm{~kg} \mathrm{~m}^{-3}$ & 1.4 \\
\hline Expansivity: surface & $\mathrm{\alpha}_{s}$ & $5.0 \times 10^{-5}$ & $\mathrm{~K}^{-1}$ & 1.0 \\
\hline Expansivity: CMB & $\alpha_{c}$ & $1.0 \times 10^{-5}$ & $\mathrm{~K}^{-1}$ & 0.20 \\
\hline Conductivity: surface & $k_{s}$ & 3.0 & $\mathrm{~W} \mathrm{~m}^{-1} \mathrm{~K}^{-1}$ & 1.0 \\
\hline Conductivity: CMB & $k_{c}$ & 6.54 & 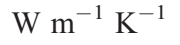 & 2.18 \\
\hline Viscosity: Surface & $\eta_{s}$ & $1.89 \times 10^{21}$ & $\mathrm{~Pa} \mathrm{~s}$ & 1.0 \\
\hline Heat capacity & $C_{p}$ & 1200 & $\mathrm{~J} \mathrm{~kg}^{-1} \mathrm{~K}^{-1}$ & 1.0 \\
\hline Depth of mantle & $D$ & 2890 & $\mathrm{~km}$ & 1.0 \\
\hline Surface temperature & $\mathrm{T}_{s}$ & 300 & $\mathrm{~K}$ & 0.12 \\
\hline \multicolumn{5}{|c|}{ Nominal Dimensional Scallings } \\
\hline Velocity & $v$ & 1 & $\mathrm{~cm} \mathrm{yr}^{-1}$ & 1468 \\
\hline Time & $t$ & 424 & Gyr & 1.0 \\
\hline Heat flux & $F$ & 2.6 & $\mathrm{~mW} \mathrm{~m} \mathrm{~m}^{-2}$ & 1.0 \\
\hline Internal heating rate & $H$ & $2.245 \times 10^{-13}$ & $\mathrm{~W} \mathrm{~kg}^{-1}$ & 1.0 \\
\hline
\end{tabular}

zaki and Karato, 2001] (with relatively low activation energy but a large activation volume term). Thus the dense material is assumed to be of lower viscosity than regular mantle by an arbitrary factor of 100 , which is probably an underestimate of a realistic contrast but numerically tractable.

\subsection{Numerics}

[8] A finite-volume multigrid method is used to solve the underlying equations, as previously described [e.g., Tackley, 1996a]. A new feature is the treatment of the chemical field, which must solve the equation

$$
\frac{\partial C}{\partial t}+\vec{v} \bullet \nabla C=0
$$

with $C$ varying from 0.0 (regular mantle) to 1.0 (dense material). $C$ is represented using tracer particles, which are present everywhere in the box and come in two types, one representing dense material and the other, regular mantle. $C$ in each grid cell is equal to the fraction of dense particles in that cell. This technique has been found (P. J. Tackley, Testing the tracer ratio nmethod for modeling active compositional fields in mantle convection simulations, submitted to Geochemistry, Geophysics, Geosystems, 2001) to be much more robust than the commonly used method [e.g., van Keken et al., 1997] of using only dense tracers and calculating $C$ from the absolute local tracer density, having the advantages of (1) avoiding nonphysical $C$ values of greater than 1.0, thereby (2) avoiding problems with numerical "settling" of tracers, and (3) requiring less tracers per cell to be reliable, with $\sim 5-10$ tracers/cell being sufficient rather than $\sim 50$ [Christensen and Hofmann, 1994].

\subsection{Domain and Boundaries}

[9] A $4 \times 4 \times 1$ Cartesian box with reflecting side boundaries is assumed. The top and bottom boundaries are impermeable and free slip and zero flux for the $C$ field. Thermally, the upper boundary is isothermal, and the lower boundary is isothermal with a temperature that is either fixed throughout the calculation or adjusted each time step to give zero net heat flow [e.g., Tackley et al., 2001]. The latter, "zero net flux isothermal" cases are less Earth-like but are included to give insight into the influence of the lower thermal boundary layer on the dynamics and observational signatures. Using a $4 \times 4 \times 1$ box with reflecting sides 
allows a doubling of horizontal grid resolution, compared to previous $8 \times 8 \times 1$ periodic models [Tackley, 1998], but the same maximum horizontal wavelength. A grid of $128 \times 128 \times 64$ cells is used, with approximately 10 million tracers used to track composition. A resolution test is given in Appendix A.

\subsection{Initial Condition}

[10] It has previously been found that chemically layered convective systems undergo a very long transient phase in which the dense layer heats up and may eventually become unstable [Davaille, 1999a, 1999b; Hansen and Yuen, 2000; Olson and Kincaid, 1991]. In order to avoid this transient phase and obtain results that are representative of the long-term behavior, the present 3-D calculations were initialized with a geotherm taken from similar two-dimensional calculations that have reached secular equilibrium. Each 2-D calculation had the same parameters as the corresponding 3-D case except that the lower layer was enforced as a fixed, impermeable boundary. Random 3-D temperature perturbations were added to this 1-D initial state.

\subsection{Cases}

[11] Three scenarios are presented: one with purely thermal convection (no dense material), one with the dense material initially taking up $10 \%$ of the mantle volume, and one with the dense material initially taking up $30 \%$ of the mantle volume. The chemical buoyancy ratio $B$ is

$$
B=\frac{\Delta \rho_{c}}{\rho_{s} \alpha_{s} \Delta T},
$$

where $\Delta \rho_{c}$ is the chemical density contrast, $\rho_{s}$ and $\alpha_{s}$ are the surface density and thermal expansivity, and $\Delta T$ is the reference temperature scale. Here, $B=0.3$ except in the zero net flux, $10 \%$ dense material case, in which it was reduced to 0.25 to increase the topography. The values of $B$ used here thus correspond to dimensional density contrasts of $150 \mathrm{~kg} / \mathrm{m}^{3}$ and $125 \mathrm{~kg} / \mathrm{m}^{3}$, respectively. These values were chosen because in an exploratory suite of 2-D calculations they gave stable layers with large topography similar to that proposed. Smaller values lead to instability, overturn, and mixing, whereas larger values lead to less layer topography. Each case was run with both CMB thermal boundary conditions.

\subsection{Filtering}

[12] In order to give a rough idea of what the results would "look" like at the resolution of current seismic tomographic models and to determine the effect of limited seismic resolution on the diagnostics considered here, a crude filtering of the model output is performed. In the horizontal directions, a (2-D) Fourier decomposition of the fields is truncated at modes with a wavelength shorter than nondimensional 0.5 (8 wavelengths across the box), a physical wavelength of $\sim 1500 \mathrm{~km}$, which, at mid mantle depth, is comparable to spherical harmonic degree $20(30,000 \mathrm{~km} / 1500 \mathrm{~km})$. Vertical smearing is performed using a sliding window seven grid cells wide, which gives a smearing of $\sim 350 \mathrm{~km}$ at the depths of interest here.

\section{Results}

[13] The present presentation is focused on the three cases with a fixed temperature isothermal lower boundary because this is more realistic for Earth. However, in order to give insight into the influence of lower boundary heat flux on the dynamics and observational signatures and because a zero flux boundary condition has commonly been used in previous mantle convection studies [e.g., Bunge et al., 1996; Houseman, 1988; Parmentier et al., 1994; Travis et al., 1990], the zero net heat flow cases are also presented but in less detail.

\subsection{Time Evolution}

[14] In the first part of the layered calculations, the initially flat layer topography increased with time. Eventually, however, the system settled into an almost equilibrium condition with peak-to-peak topography remaining roughly constant and only a slow evolution of the convective pattern and slow entrainment of the dense material. Between the initial growth and long-term stable phases was an "overshoot" phase in which the layer topography exceeded its long-term stable value, resulting in a 

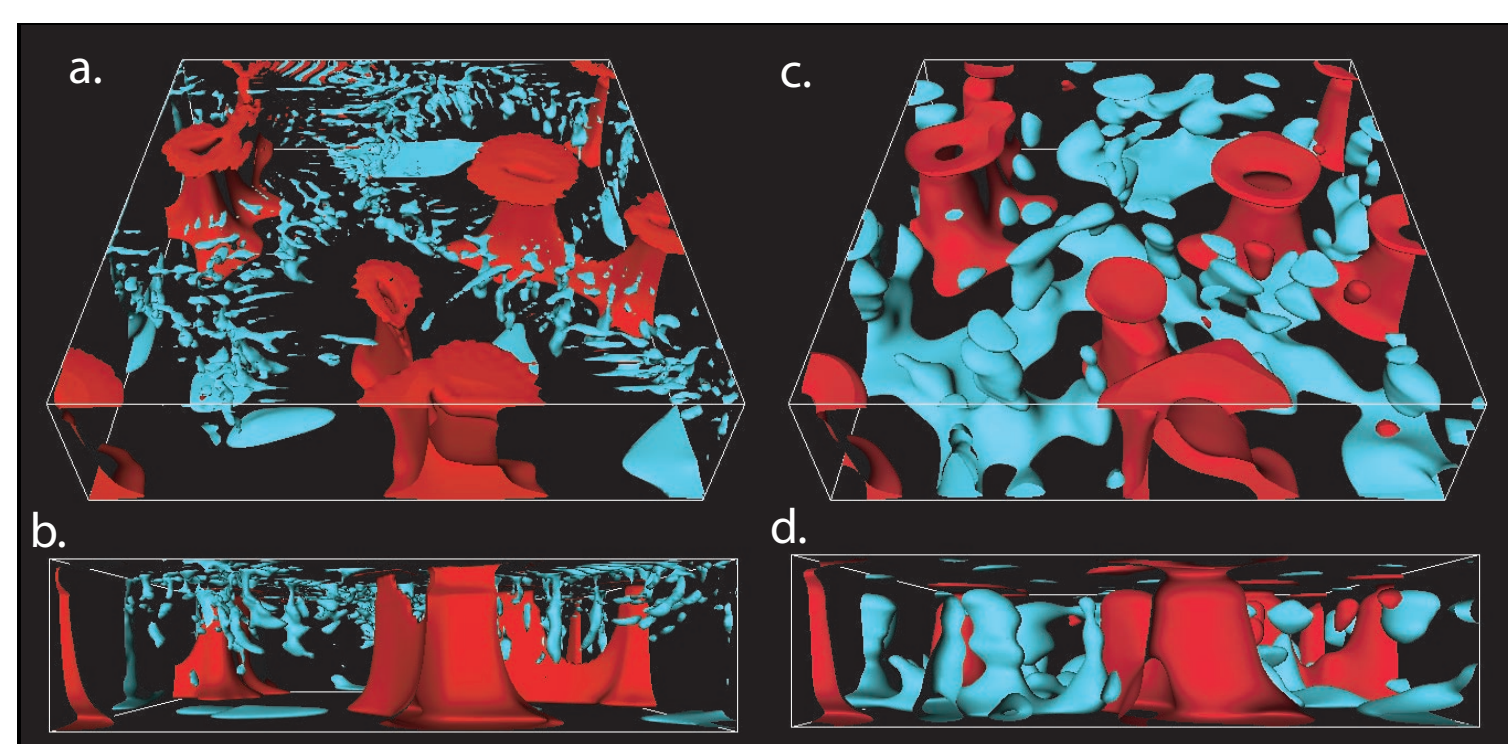

d.
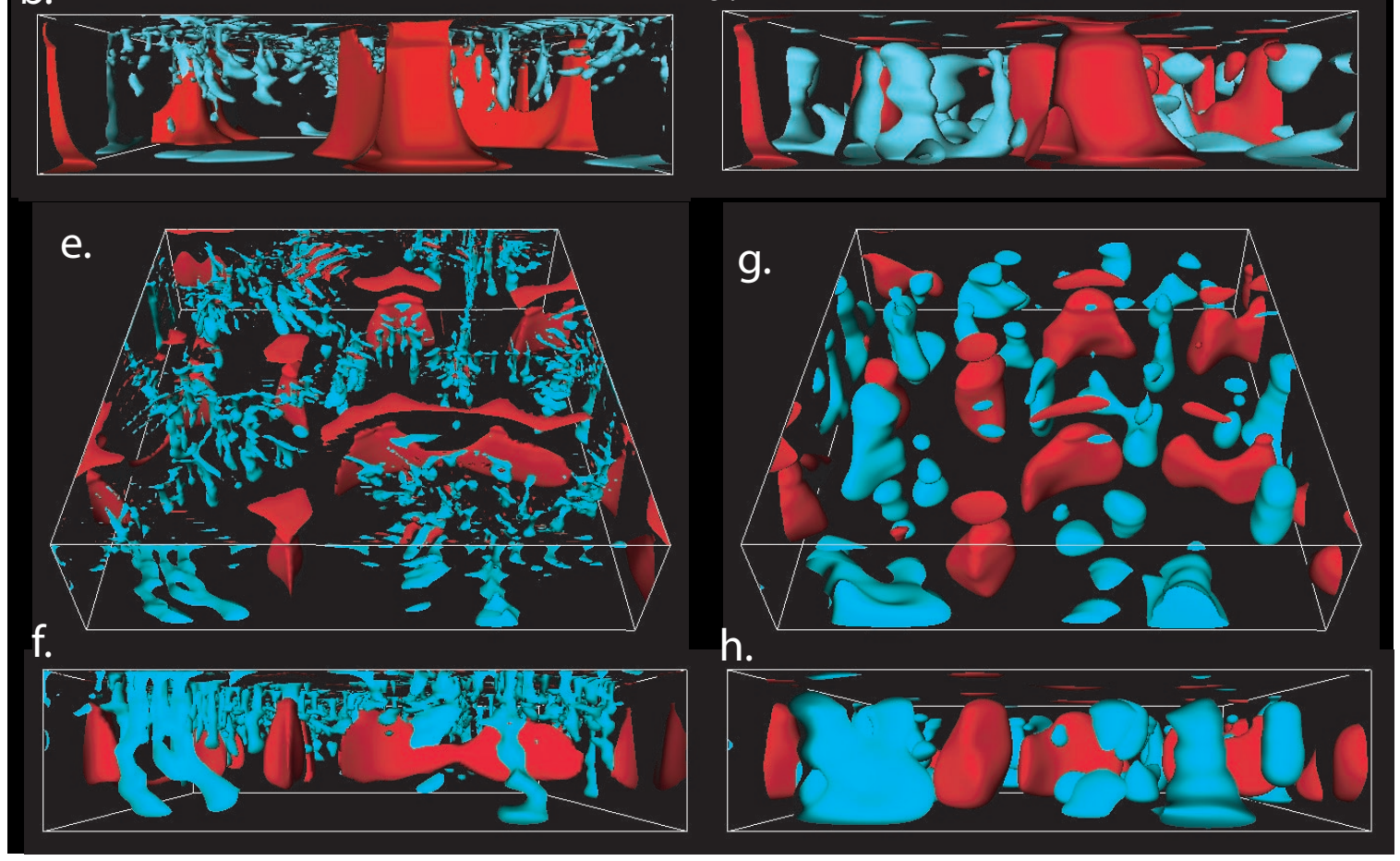

Figure 1. Isosurfaces of residual temperature for the two isochemical cases with (a)-(d) a fixed temperature CMB and (e) $-(\mathrm{h})$ a zero net flux CMB. The left column fields (Figures 1a, 1b, 1e, and 1f) are unfiltered, whereas the right column fields (Figures 1c, 1d, 1g, and 1h.) are filtered. Isosurfaces values (red being hot) are \pm 0.05 (Figures 1a and 1b), \pm 0.025 (Figures $1 \mathrm{c}$ and $1 \mathrm{~d}$ ), \pm 0.075 (Figures $1 \mathrm{e}$ and 1f), and +0.05 (red) and -0.04 (blue) (Figures $1 \mathrm{~g}$ and $1 \mathrm{~h}$ ).

pulse of entrainment. The long-term stable phase was reached in $\sim 8000-12,000$ time steps, corresponding to nondimensional times of $\sim 4-5 \times 10^{-3}$ ( $\sim 1.7-2.1$ billion years). The frames analyzed here correspond to an early part of the long-term phase. Calculations were run for 20,000 time steps.

\subsection{Isochemical Convection}

[15] The isochemical cases at a time of 0.0105 $(\sim 4.5$ Gyr) for the fixed $T \mathrm{CMB}$ or 0.02025
( $~ 8.6$ Gyr) for the zero net heat flow CMB are illustrated using isosurfaces of residual temperature (i.e., temperature relative to geotherm) (Figures 1a, $1 \mathrm{~b}, 1 \mathrm{e}$, and 1f) and slices of the superadiabatic temperature field (Figure 2). The case with a fixed temperature $\mathrm{CMB}$ is $\sim 50 \%$ heated from below and displays strong, broad, hot plume-like upwellings and time-dependent downwellings that are linear but with much small-scale complexity, similar to previous, strongly basally heated results [Balachandar et al., 1992, 1995]. The zero net 

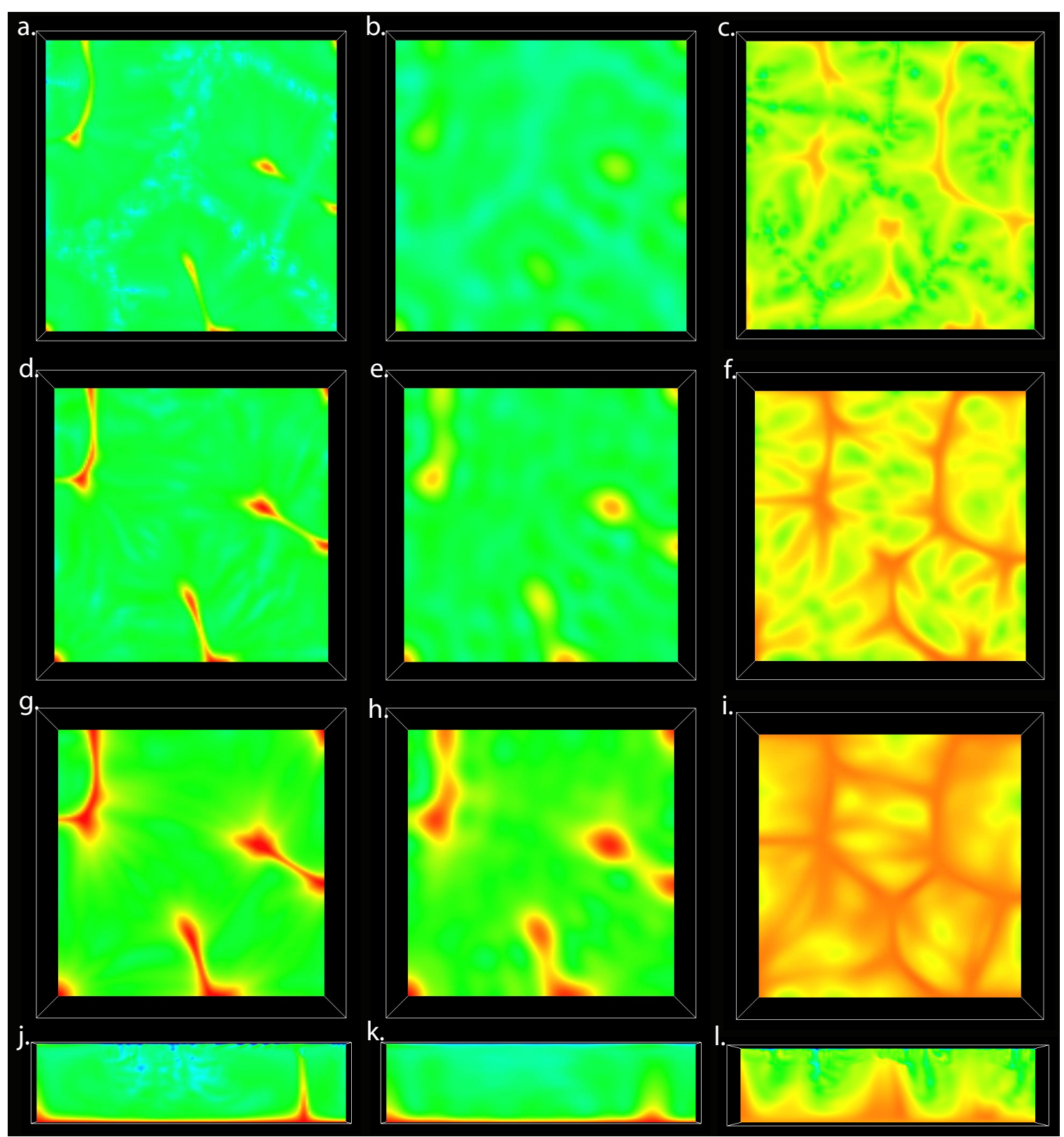

Figure 2. (a)-(i) Horizontal and (j)-(l) vertical slices through the superadiabatic temperature field for the two isochemical cases. The fixed CMB case is plotted with a temperature scale of $0-1$ in the left column (Figures $2 \mathrm{a}, 2 \mathrm{~d}$, $2 \mathrm{~g}$, and $2 \mathrm{j}$ ) with full (unfiltered) field and the center column (Figures $2 \mathrm{~b}, 2 \mathrm{e}, 2 \mathrm{~h}$, and $2 \mathrm{k}$ ) with the filtered field. The left column (Figures 2c, 2f, 2i, and 2l) shows the full field for the zero net flux CMB case with a temperature scale of 0-0.5. Horizontal slices are at $1850 \mathrm{~km}$ (Figures 2a-2c), $770 \mathrm{~km}$ (Figures 2d-2f), and $270 \mathrm{~km}$ (Figures 2g-2i) above the CMB.

CMB flux case, although 100\% heated from within, looks quite different from internally heated convection with constant properties [e.g., Houseman, 1988; Parmentier and Sotin, 2000; Tackley, 1996b] or even with depth-dependent properties but constant viscosity [Bercovici et al., 1989a; Schubert, 1992], in which the convection is domi- nated by very time-dependent, closely spaced cylindrical downwellings. Instead, this case displays quite widely spaced downwellings that are linear but have much small-scale complexity. This difference is due mostly to the depth-dependence of viscosity as well as other properties [Bunge et al., 1996; Tackley, 1996a]. 
[16] Regardless of heating mode, many of the downwellings do not penetrate to the CMB (Figures 1b, 1f, $2 \mathrm{j}$ and $2 \mathrm{l}$ ), even though there is no particular feature (e.g., phase change or chemical layer) to stop them, as previously noted in 2-D simulations [van Keken and Ballentine, 1999]. However, when the residual temperature is filtered to seimic tomographic wavelengths (Figures $1 \mathrm{~d}$ and $1 \mathrm{~h}$ ), downwellings appear to penetrate to the $\mathrm{CMB}$, particularly with significant basal heating (Figure 1d). Thus while it may be tempting to interpret these models as an explanation for the apparent nonpenetration of some slabs in Earth to the CMB (observed in some seismic tomographic models, e.g., van der Hilst and Karason [1999]), caution must be exercised.

[17] In the zero net CMB flux case, linear hot upwellings are visible in the midmantle. It is interesting that such features, which are associated with the "passive" return flow to the "active" cold downwellings, are possible even with no basal heating, because it has previously been argued that they are observed in seismic tomography models deep under spreading centers [Su et al., 1992]. The present results suggest that the seismically observed features are not necessarily associated with active hot upwellings from the CMB. Indeed, where such active features are present (as in the fixed $T$ CMB case), they tend to be plume-like, although this may be changed by plate tectonics, which is lacking in these models.

[18] To compare the convective vigor to Earth's, it is useful to consider the surface velocity, which, for the fixed $T$ CMB case, is $3200(2.2 \mathrm{~cm} / \mathrm{yr}) \mathrm{rms}$ and $8400(5.7 \mathrm{~cm} / \mathrm{yr})$ peak of the same magnitude, though slightly lower, than Earth's plate velocities. The volume-averaged rms velocity is much lower at $1070(0.73 \mathrm{~cm} / \mathrm{yr})$ because flow is relatively sluggish in the high viscosity lower mantle. The velocities are slightly lower in the zero flux CMB case.

\subsection{Global, Thick (30\%) Layer}

[19] Full resolution $C$ (Figures 3a, 3b, 3i and 3j) and $T$ (Figures 3 c. $3 \mathrm{~d}, 3 \mathrm{k}$, and 31 and Figures $4 \mathrm{a}-\mathrm{d}$ and $4 \mathrm{q}$ ) fields are shown at an elapsed time of $6.64 \times 10^{-3}(2.8 \mathrm{Gyr})$ for the fixed $T$ CMB case and $4.71 \times 10^{-3}(2 \mathrm{Gyr})$ for the zero net flux case. Surface velocities are similar to the isochemical case at $\sim 2500(1.7 \mathrm{~cm} / \mathrm{yr}) \mathrm{rms}$ and $\sim 7000(4.8 \mathrm{~cm} / \mathrm{yr})$ peak. The CMB heat flux is $\sim 0$ for both CMB boundary conditions and fluctuates around $\sim 25$ at the surface, and so the cases are very similar. The dense material is swept around by the convection, being thinned under downwellings and thickened into ridges under upwellings, similar to laboratory results [Olson and Kincaid, 1991], and displaying a peak-topeak undulation of $\sim 800-1000 \mathrm{~km}$, similar to the model proposed by [Kellogg et al., 1999]. Entrainment, which gradually depletes the layer, occurs along the ridges. The dense material is much hotter than the overlying regular mantle, so that a horizontal slice at $770 \mathrm{~km}$ above the CMB (Figure $4 \mathrm{~b}$ ) displays substantial temperature heterogeneity. Small-scale convection occurs inside this hot, low-viscosity, dense material (Figures $4 b-4 d)$. Hot linear upwellings arise from the top of the dense layer (Figures 3c, 3d and 4a) and rise as sheets to the upper mantle where they partially break up. Downwellings are basically linear but with much time-dependent small-scale structure and are weak features in the $T$ field compared to deep mantle structure. The filtered fields (Figures $3 \mathrm{e}-3 \mathrm{~h}$ and Figure $4 \mathrm{e}-4 \mathrm{~h}$ ) reproduce the large-scale major deep mantle structures well, but small-scale structures in the upper mantle and inside the dense layer are lost.

[20] Perhaps one of the most remarkable features in the $T$ field is the lack of heterogeneity inside the dense layer (Figure 3d, 3h, and 31), which arises because its low viscosity (which arises physically because it is very hot) allows it to convect vigorously with relatively small temperature contrasts. (Since $\Delta T$ for internally heated convection scales as $\eta^{1 / 4}$ with other parameters fixed [e.g., Tackley, 2001], $\Delta T$ is reduced by a factor of $\sim 3.1$ by the lower viscosity prescribed here; furthermore since velocity scales as $\eta^{-1 / 2}$, it is increased by a factor of $\sim 10$ ). This lack of deep mantle heterogeneity contrasts sharply with the strong middle lower mantle heterogeneity. 

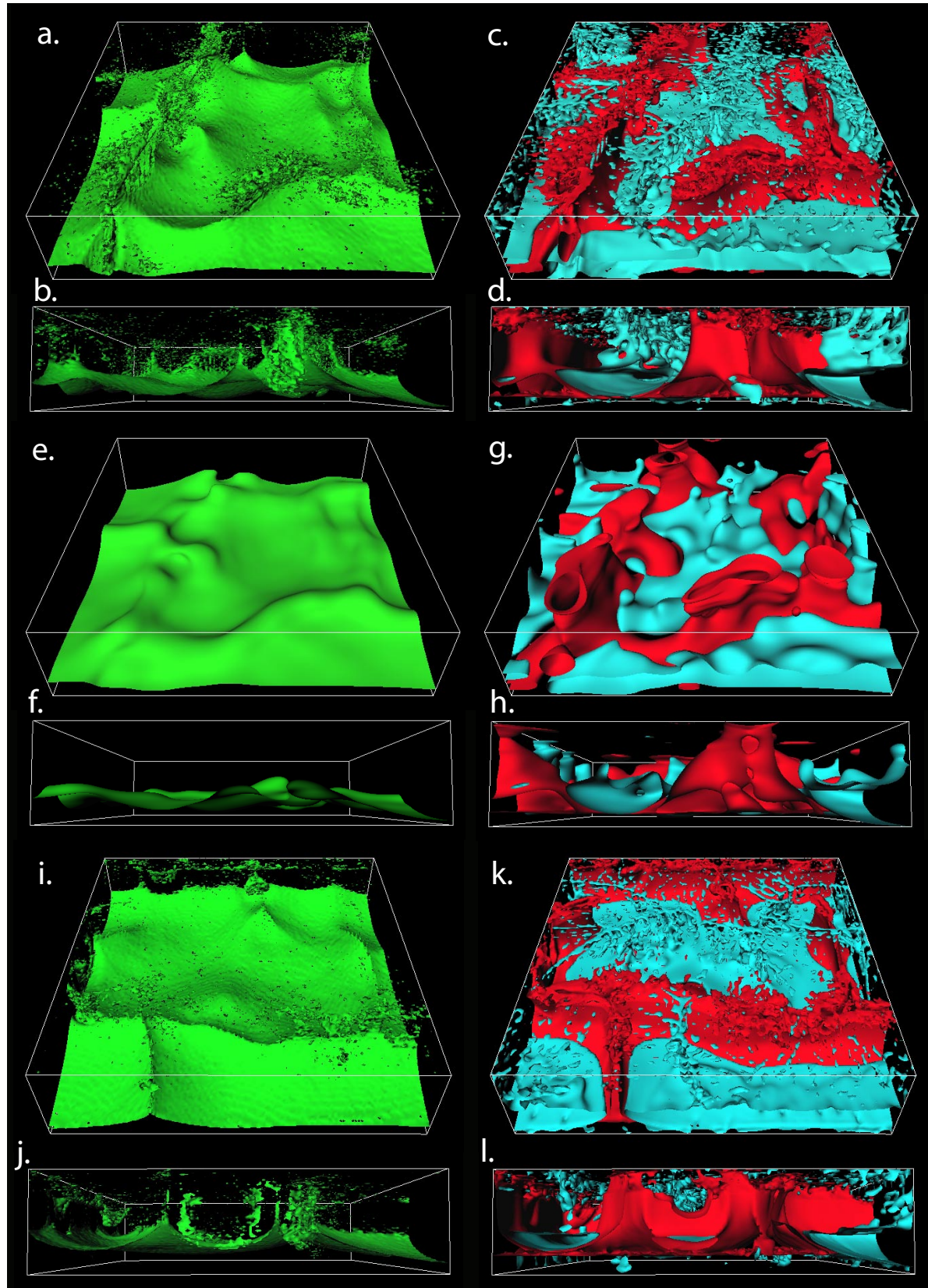

Figure 3. Isosurfaces of $C$ (left column) and residual $T$ (right column) fields for the two cases with a global layer (30\% dense material). For the fixed CMB case both full (Figures 3a-3d) and filtered (Figures 3e-3h) fields are plotted whereas for the zero net flux CMB case only the full fields are plotted (Figures 3i-31). The contour level is $C=0.5$ (green) for all compositional plots, and for the temperature plots the contour levels are $T= \pm 0.075$ (Figures $3 \mathrm{c}, 3 \mathrm{~d}, 3 \mathrm{k}$, and $3 \mathrm{l}$ ), $T= \pm 0.0375$ (Figures $3 \mathrm{~g}$ and $3 \mathrm{~h}$ ).

\subsubsection{Heterogeneity maps}

[21] The heterogeneity and its dependence on horizontal wavelength can be quantified using spectral heterogeneity maps (SHM [Tackley et al., 1994]), which are contour plots of the rms horizontal spatial frequency spectrum as a function of depth (Figure 5).
For best comparison with seismic tomography, SHMs for filtered fields are plotted, although the filtering has little effect on this diagnostic.

[22] The temperature SHM for the isochemical, fixed $T$ CMB case (Figure 5a) displays a peak in the upper boundary layer and a larger peak in the 


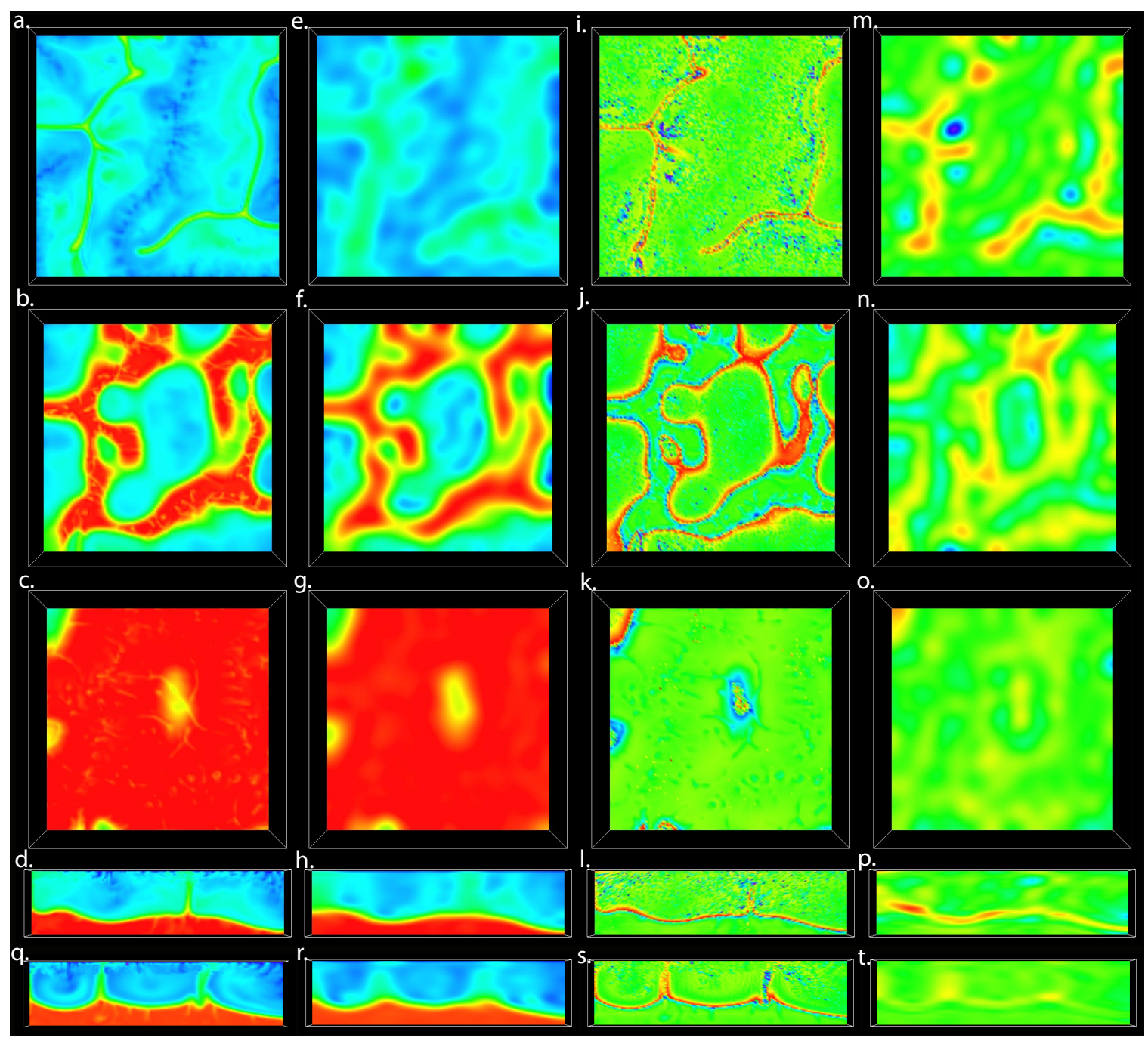

Figure 4. Slices through the fields for cases with a global layer $(30 \%$ dense material) and (a)-(o) a fixed temperature CMB, (q)-(t) a zero net flux CMB. Superadiabatic temperature (Figures $4 \mathrm{a}-4 \mathrm{~d}$ and $4 \mathrm{q}$ ), filtered superadiabatic temperature (Figures $4 \mathrm{e}-4 \mathrm{~h}$ and $4 \mathrm{r}$ ), seismic velocity (Figures $4 \mathrm{i}-4 \mathrm{l}$ and $4 \mathrm{~s}$ ), and filtered seismic velocity (Figures $4 \mathrm{~m}-4 \mathrm{p}$ and $4 \mathrm{t}$ ). Horizontal slices are at $1850 \mathrm{~km}$ (Figures 4a, 4e, 4i, and 4m), $770 \mathrm{~km}$ (Figures 4b, $4 \mathrm{f}, 4 \mathrm{j}$, and $4 \mathrm{n}$ ), and $270 \mathrm{~km}$ (Figures $4 \mathrm{c}, 4 \mathrm{~g}, 4 \mathrm{k}$, and $4 \mathrm{o}$ ) above the CMB.

lower thermal boundary layer. With no net CMB heat flow (Figure $5 \mathrm{c}$ ), the lower boundary layer peak is lost and heterogeneity is more constant in the lower mantle.

[23] In contrast, the temperature SHM for the 30\% case (Figure 5k) displays a huge "bulls eye" in the middle lower mantle region (corresponding to the region where the dense layer undulates). The $C$ field heterogeneity (Figure 51) is very similar.

[24] SHMs for seismic tomographic models of [Masters et al., 2000] and an updated version of the model by S. P. Grand [Grand et al., 1997]
(Figure 5d and 5e) do not display such a feature but rather a strong peak at the top of the mantle and a lesser peak near the CMB. The strong peak at the top of the mantle is likely amplified by the presence of continents [Gurnis and Zhong, 1991; Tackley, 2000c], which are lacking in this model; hence only lower mantle signatures can be reasonably compared in this study.

\subsubsection{Combining $T$ and $C$}

[25] Because seismic velocity depends on both temperature and composition, fields which have some similarity, it is reasonable to hypothesize that 

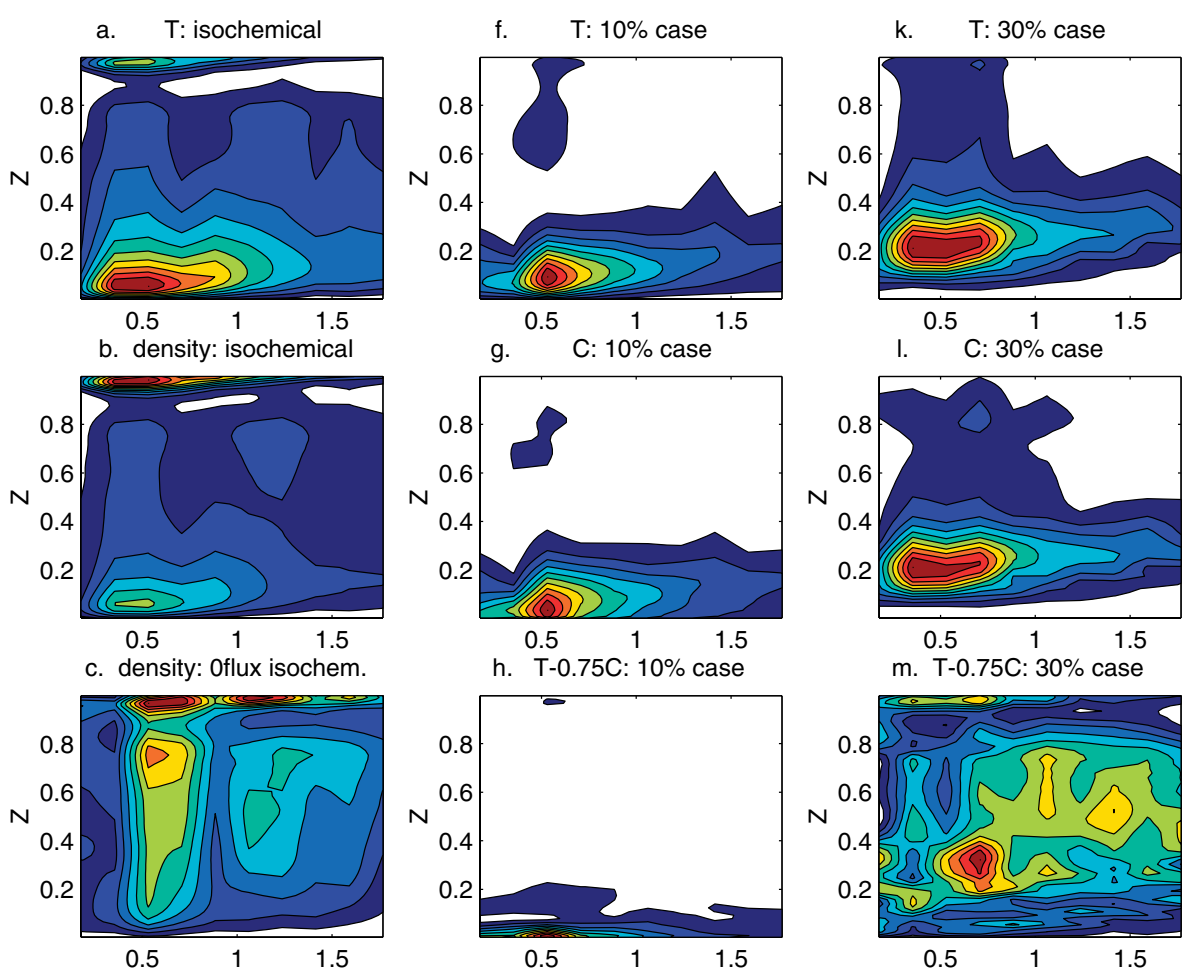

h. T-0.75C: $10 \%$ case
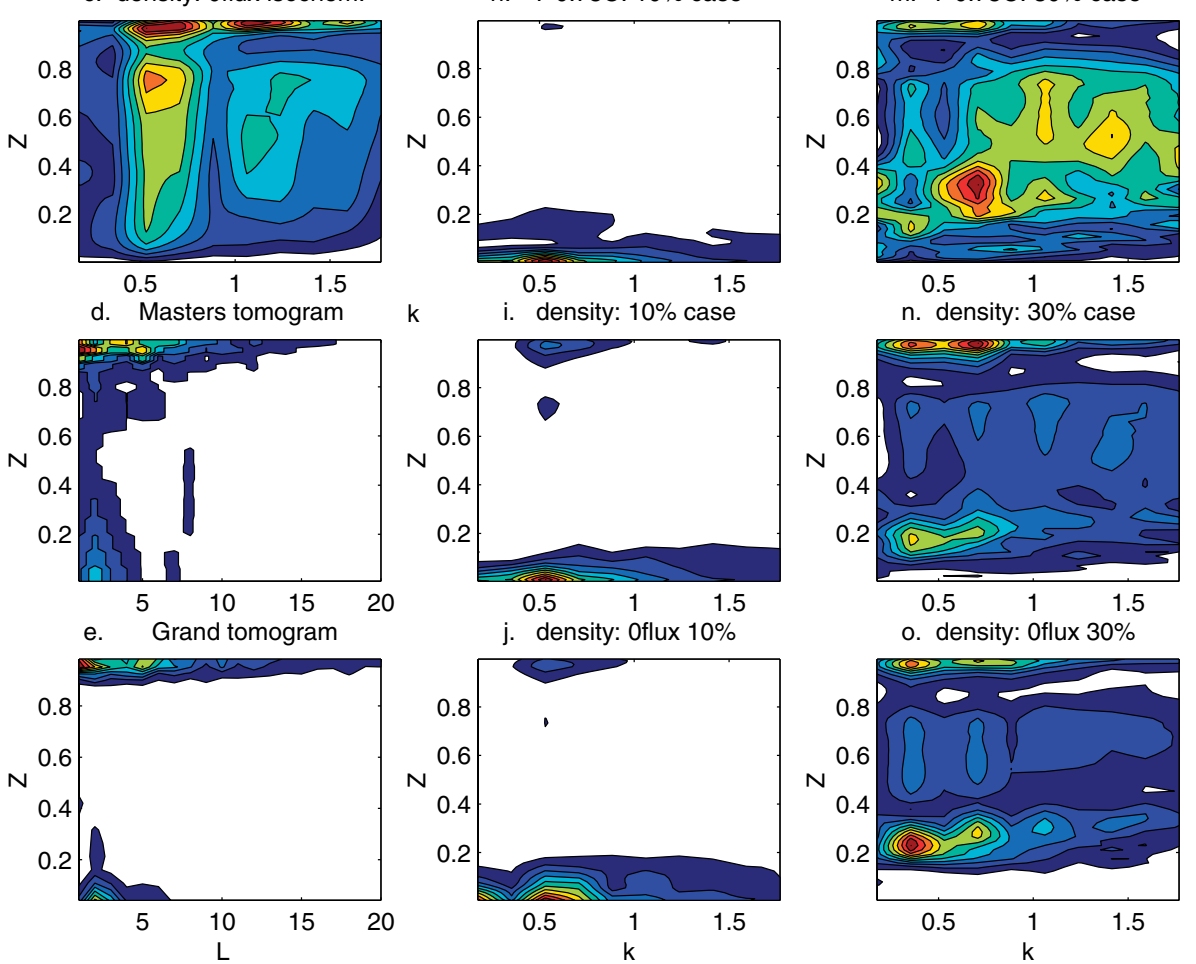

Figure 5. Spectral heterogeneity maps for filtered fields of presented cases. Isochemical: (a) temperature and (b) density for the the fixed CMB case and (c) density for the isochemical zero net flux CMB case. Seismic tomography: (d) seismic tomogram of [Masters et al., 2000]; (e) seismic tomogram of S. P. Grand (updated from [Grand et al., 1997]). Models with $10 \%$ dense material: (f) temperature (g) composition (h) $T-0.75 \mathrm{C}$ and (i) density for the case a fixed $T$ CMB and (j) density for the case with a zero net flux CMB. Models with $30 \%$ dense material: $(\mathrm{k})$ temperature (l) composition (m) $T-0.75 \mathrm{C}$ and (n) density for the fixed $T$ CMB case, and (o.) density for the model with a zero net flux CMB. Contour levels are normalized to the maximum amplitude in each plot, which is 0.0566 (Figure 5a), 0.0310 (Figure 5b), 0.0106 (Figure 5c), 0.0268 (Figure 5d), 0.0296 (Figure 5e), 0.2139 (Figure 5f), 0.2395 (Figure 5g), 0.1552 (Figure 5h), 0.0685 (Figure 5i), 0.0736 (Figure 5j), 0.1537 (Figure 5k), 0.1642 (Figure 5l), 0.0226 (Figure 5m), 0.0217 (Figure 5n), and 0.0265 (Figure 5o). 
the effects of $T$ and $C$ on $V$ might largely cancel out, leading to a very subdued signature for the dense layer. To test this, two methods of combining these fields are used here. The first method is a straightforward linear combination of the fields:

$$
\Delta V \propto \Delta T+a \Delta C,
$$

where $\Delta V, \Delta T$, and $\Delta \mathrm{C}$ are the velocity, temperature, and composition anomalies relative to the horizontal mean and $a$ is a constant that is adjusted to minimize the seismic signature of the layer. For the fixed $T$ CMB case, it was found that $\mathrm{a} \approx-0.75$ provides the best cancellation of the fields. The seismic velocity field for this value is plotted in Figures $4 i-41$. Slices that sample both the dense layer and regular mantle (Figures $4 j$ and 41 ) confirm that the dense layer has roughly the same seismic velocity anomaly as the bulk of the mantle for this value of $a$ but that there is a strong signature from the boundary layer between the two layers and from the upwellings that arise from the top of the layer (Figures $4 \mathrm{i}$ and 41). The boundary layer signature arises because the thermal boundary layer is conductive, thus extending $\sim 100 \mathrm{~km}$ on either side of the boundary, whereas the compositional boundary is sharp. After filtering (Figure $4 m-4 p$ ) the boundary layer signature becomes indistinct and "blob"-like due to the near cancellation of the positive and negative bands at long wavelength. However, strong signatures from the induced upwellings remain (Figure 4m).

[26] SHMs for the combined fields (Figures 5m) indicate that while the midmantle heterogeneity is strongly suppressed, with a peak amplitude of 0.0226 compared to 0.1537 for the $T$ field, it is still the dominant heterogeneity in the mantle. The peak has moved upward, being influenced more by the induced upwellings than the undulating layer boundary. SHMs have been computed for a wide range of $a$ values and indicate that this signature is robust: it is not possible to completely eliminate the strong layer signature by a linear combination of the $T$ and $C$ fields.

[27] A second combination of the fields is the density anomaly, given by

$$
\Delta \rho=\rho(z) \alpha(z) \Delta T+B \Delta C,
$$

where $\rho(z)$ and $\alpha(z)$ are the reference state density and thermal expansivity, respectively. This is expected to be neutral for large-scale stable (i.e., nonmoving) features, otherwise they would be moving. The density SHM for the $30 \%$ case (Figure 5h) also indicates a strong signature from the dense layer boundary, but the upper boundary signature also becomes visible.

\subsection{Discontinuous Thin (10\%) Dense Layer}

[28] The full resolution $C$ (Figures $6 \mathrm{a}, 6 \mathrm{~b}, 6 \mathrm{i}$, and $6 \mathrm{j}$ ) and $T$ (Figures $6 \mathrm{c}, 6 \mathrm{~d}, 6 \mathrm{k}, 6 \mathrm{l}$ and Figures $7 \mathrm{a}-\mathrm{d}$, and $7 \mathrm{q})$ fields are shown at a time of $4.4 \times 10^{-3}(1.9 \mathrm{Gyr})$ for the fixed $T$ CMB case and $6.3 \times 10^{-3}(2.7 \mathrm{Gyr})$ for the zero net flux CMB case and show that the dense material is swept into linear "piles" of material, exposing large areas of the CMB. Entrainment occurs from the peaks of these ridges. The dense material is substantially hotter than the regular mantle (Figures $7 d$ and $7 q$ ), so that a horizontal slice $270 \mathrm{~km}$ above the CMB (Figure 7c) displays substantial temperature heterogeneity. In the zero net flux case, the temperature of the hot material is lower. Some small-scale convection is visible inside the hot, dense, low-viscosity material (Figure 7c). Hot linear upwellings arise from the top of the dense material (Figures 6c, 6d, 6k, and 61 and Figures $7 a-7 b$ ), rising as sheets into the upper mantle. Downwellings have an almost linear alignment but with much time-dependent small-scale structure. The residual temperature field (Figures $6 \mathrm{c}, 6 \mathrm{~d}, 6 \mathrm{k}$, and 61) shows large pools of apparently cold material directly above the CMB; however, examination of the absolute $T$ field at this depth (Figures $7 \mathrm{c}, 7 \mathrm{~d}$, and 7q) indicates that these are not colder than the overlying mantle but rather just cold compared to the exceptionally hot dense material. Filtered versions of these fields (Figures $6 \mathrm{e}-6 \mathrm{~h}$ and Figures $7 \mathrm{e}-7 \mathrm{~h})$ indicate that the major deep mantle structures remain intact but that much of the small-scale upper mantle structure is lost. The main effect of CMB heat flux is to make the piles of dense material hotter and to strengthen the upwellings arising from them. Upwellings do not form away from the dense piles even with substantial CMB heat flux.

[29] Combining the fields, it was found that $a \approx$ -0.75 minimizes the seismic signature for the fixed 

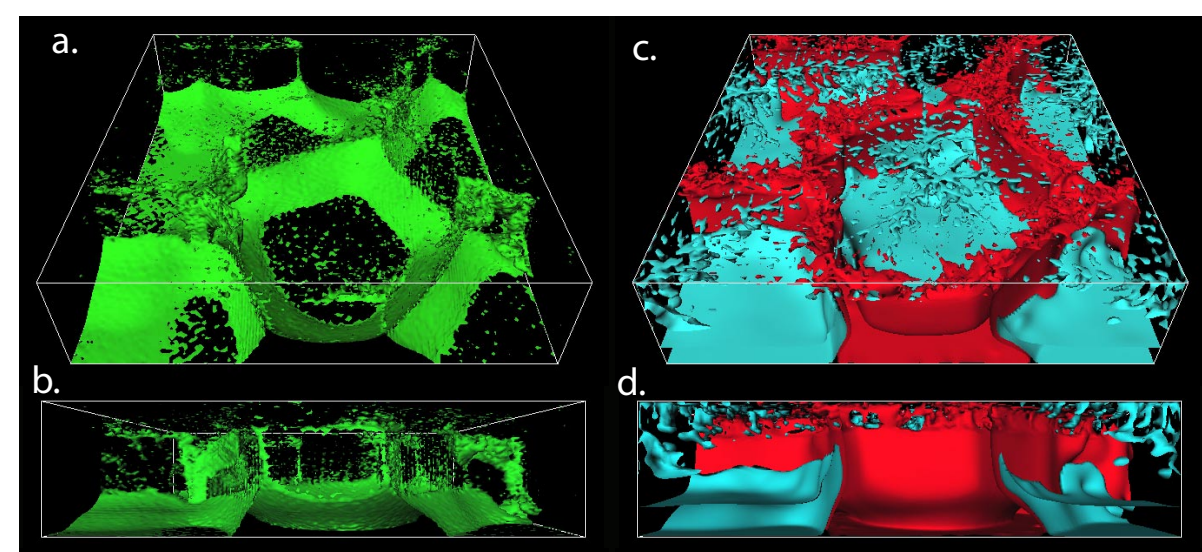

d.
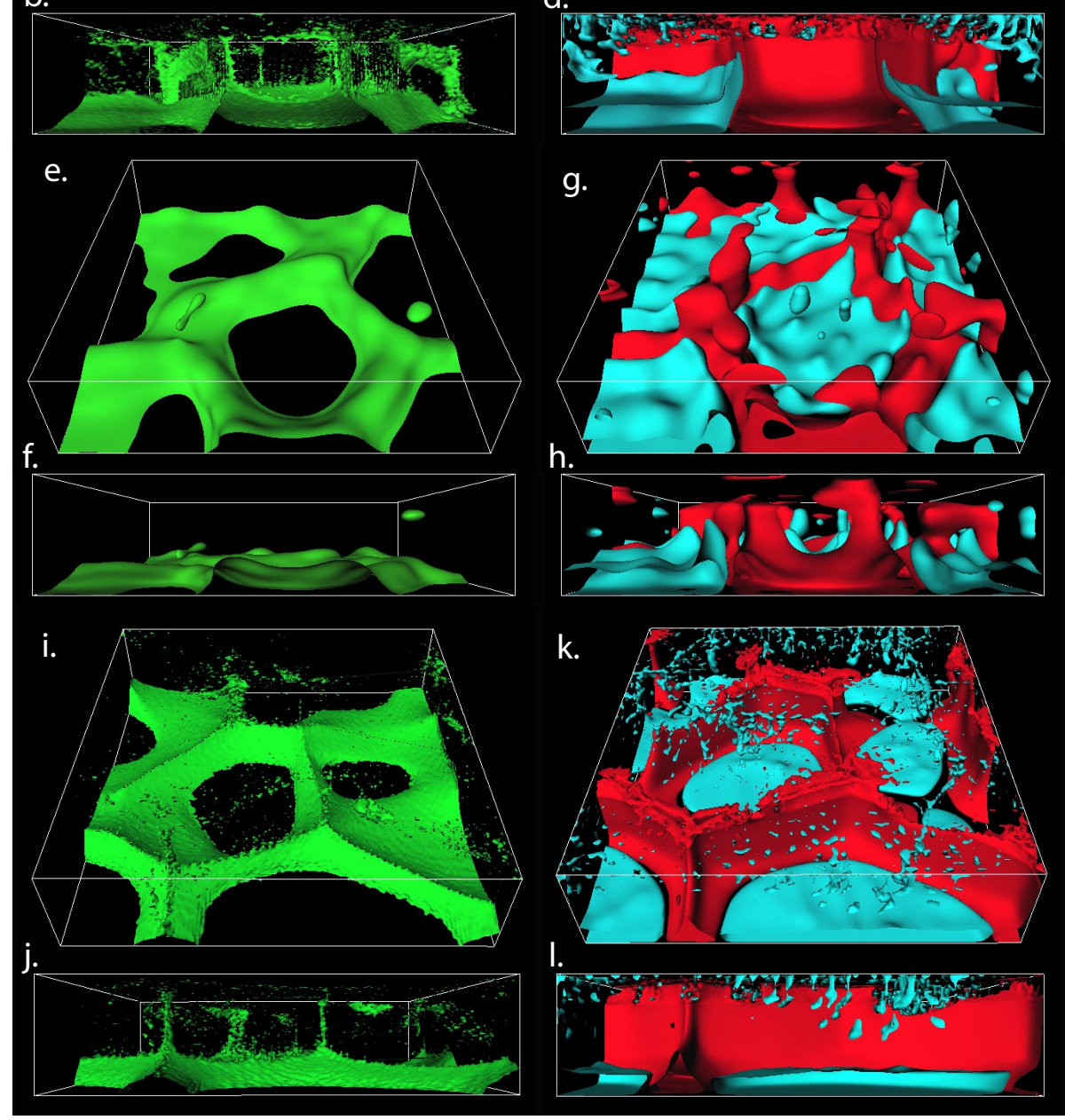

Figure 6. Isosurfaces of $C$ (left column) and residual $T$ (right column) fields for the two cases with a discontinuous layer (10\% dense material). For the fixed CMB case both full (Figures $6 \mathrm{a}-6 \mathrm{~d}$ ) and filtered (Figures $6 \mathrm{e}-6 \mathrm{~h}$ ) fields are plotted whereas for the zero net flux CMB case only the full fields are plotted (Figures 6i-61). The contour level is $C=0.5$ (green) for all compositional plots, and for the temperature plots the contour levels are $T= \pm 0.075$ (Figures $6 \mathrm{c}, 6 \mathrm{~d}, 6 \mathrm{k}$, and 61) and $T= \pm 0.0375$ (Figures $6 \mathrm{~g}$ and $6 \mathrm{~h}$ ).

CMB $T$ case. The dominant features in the seismic velocity field (Figures $7 \mathrm{i}-71$ and $7 \mathrm{~s}$ ) are again the thermochemical boundary layer and the hot linear upwellings from the top of the piles. Just above the $\mathrm{CMB}$, strong heterogeneity associated with the thermal boundary layer is visible. Upon filtering (Figures $7 \mathrm{~m}-7 \mathrm{p}$ and $7 \mathrm{t}$ ), the boundary layer becomes indistinct and blobby while the upwellings remain clear.

[30] Spectral heterogeneity maps display a strong heterogeneity "bulls eye" in both $T$ and $C$ fields (Figures $5 \mathrm{f}$ and $5 \mathrm{~g}$ ), but this is now focused just above the $\mathrm{CMB}$ rather than in the middle lower 


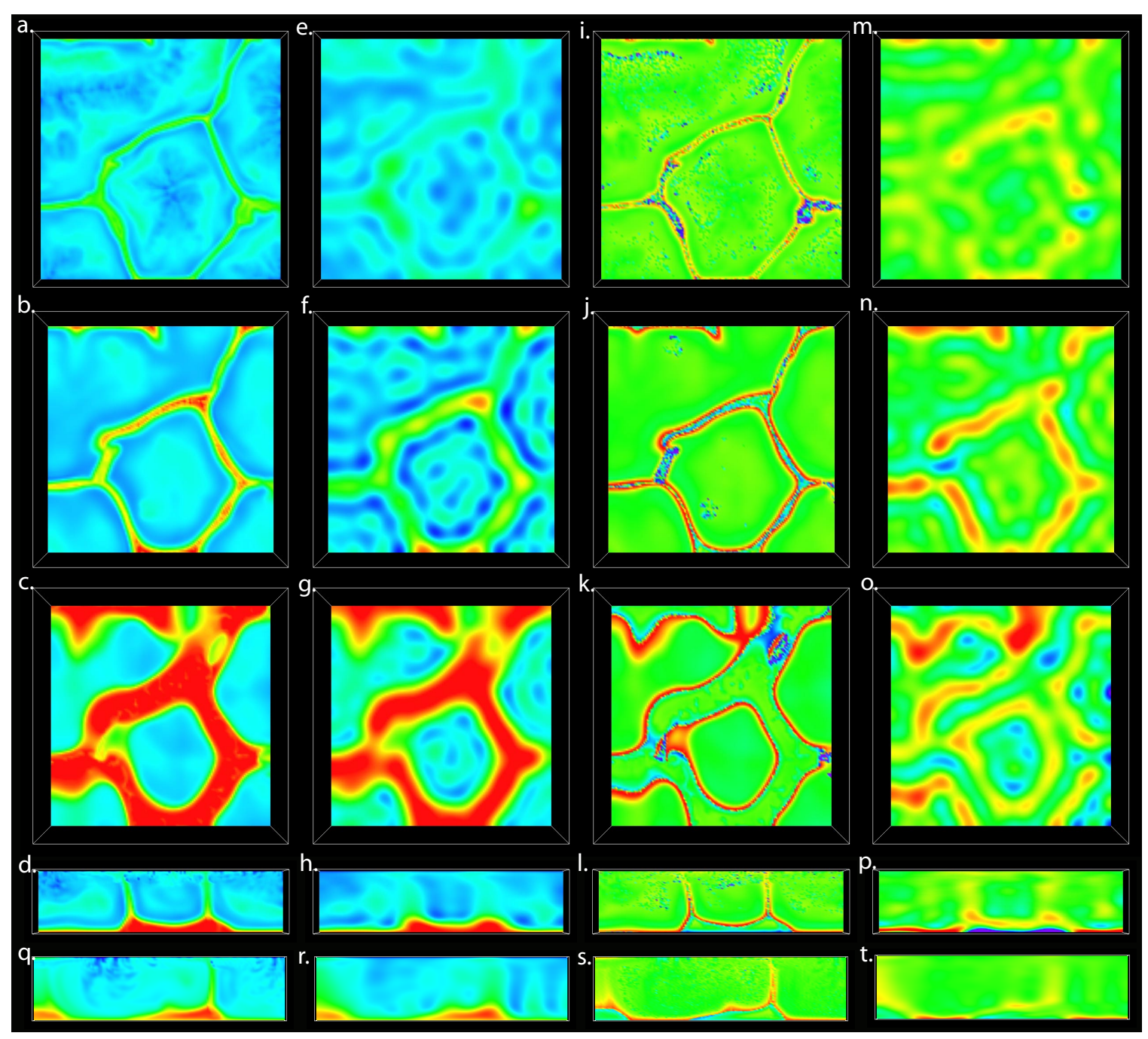

Figure 7. Slices through the fields for cases with a discontinuous layer (10\% dense material) and (a)-(o) a fixed temperature CMB, (q)-(t) a zero net flux CMB. Superadiabatic temperature (Figures $7 \mathrm{a}-7 \mathrm{~d}$ and $7 \mathrm{q}$ ), filtered superadiabatic temperature (Figures $7 \mathrm{e}-7 \mathrm{~h}$ and $7 \mathrm{r}$ ), seismic velocity (Figures $7 \mathrm{i}-71$ and $7 \mathrm{~s}$ ), and filtered seismic velocity (Figures $7 \mathrm{~m}-7 \mathrm{p}$ and $7 \mathrm{t}$ ). Horizontal slices are at $1850 \mathrm{~km}$ (Figures 7a, 7e, 7i, and 7m), $770 \mathrm{~km}$ (Figures 7b, $7 \mathrm{f}, 7 \mathrm{j}$, and $7 \mathrm{n}$ ), and $270 \mathrm{~km}$ (Figures $7 \mathrm{c}, 7 \mathrm{~g}, 7 \mathrm{k}$, and $7 \mathrm{o}$ ) above the CMB.

mantle. Indeed, the $T$ field has zero lateral heterogeneity at the CMB owing to the isothermal boundary condition. This peak is greatly reduced and pushed toward the CMB in the SHMs of both seismic velocity (Figure 5h) and density (Figure 5i), with a faint upper boundary peak emerging. These model SHMs compare reasonably well to those for actual seismic tomographic models (Figures $5 \mathrm{~d}$ and $5 \mathrm{e}$ ) except that the horizontal wavelength is too short and there is less signal from the upper boundary layer, which is expected because the models lack continents.
[31] The near-surface convective vigor is slightly higher than the other cases, with an rms surface velocity of $2800(1.9 \mathrm{~cm} / \mathrm{yr})$ and a peak surface velocity of $9300(6.3 \mathrm{~cm} / \mathrm{yr})$.

\subsection{Temperature Profiles}

[32] The high temperature of the deep layer, which arises because its strong internal heating must be conducted across the chemical interface, has a major effect on the geotherm for both lower boundary conditions (Figure 8). For isochemical convection, 
a. $\quad$ Fixed $\mathrm{T} \mathrm{CMB}$

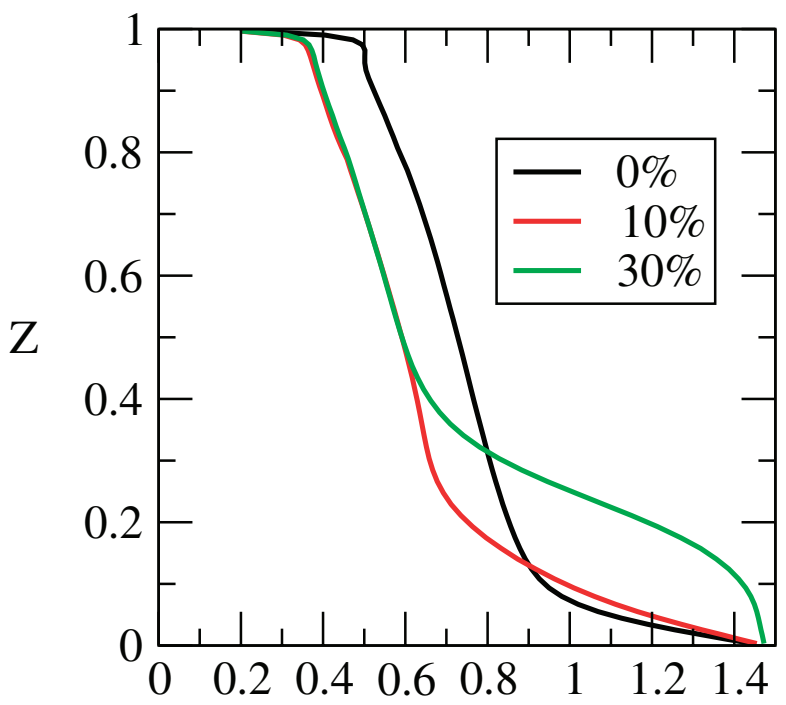

Temperature b. Zero net flux CMB

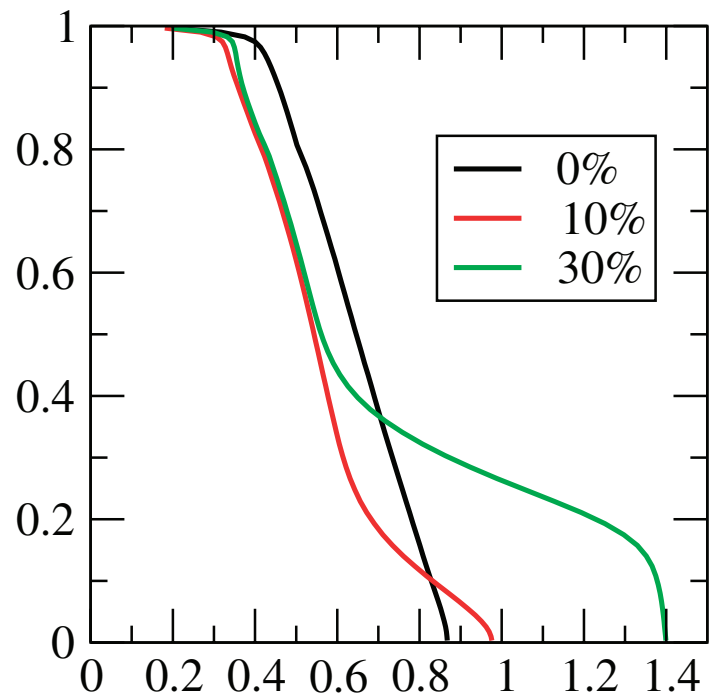

Temperature

Figure 8. Geotherms for the illustrated frames of (a) the three fixed $T$ CMB cases and (b) the three zero net flux $\mathrm{CMB}$ cases. Isochemical (black line), 10\% dense material (red line), and 30\% dense material (green line).

the geotherm is expected to be approximately adiabatic in the interior (slightly sub adiabatic for internally heated convection [Parmentier et al., 1994]), and the present isochemical cases are consistent with this (Figure 8 black lines). With a global dense layer (green lines), the upper mantle geotherms are slightly lower (owing to downward focusing of heat producing elements into the dense layer), but the deep mantle geotherms are substantially higher such that the zero net flux case rises to almost the same value as the fixed CMB case, consistent with the latter having very low CMB heat flux.

[33] The high deep mantle temperatures in the globally layered case may cause certain problems. The CMB heat flux is very low, possibly sometimes negative (consistent with thermal evolution calculations [Schubert and Spohn, 1981; McNamara and van Keken, 2000]), which may be insufficient to drive the geodynamo [e.g. Labrosse et al., 1997]. Additionally, it is conceivable that deep mantle temperatures could get hot enough for melting [Zerr et al., 1998]: although the present nominal scalings (Table 1) lead to a CMB temperature of only $3700 \mathrm{~K}$, a scaling based on matching upper mantle temperatures could lead to a higher CMB value; furthermore spherical geometry may necessitate higher deep mantle temperatures to accomplish the same total heat loss from the radiogenically enriched layer. More research needs to be done on this issue.

\subsection{Radial Correlation Functions}

[34] The radial correlation function (RCF) is a normalized cross correlation of one horizontal slice with another [Jordan et al., 1993]. Here RCFs for filtered and unfiltered $T$ and seismic velocity fields are considered. It may be helpful to recall that RCFs have a value of unity across the diagonal, corresponding to the perfect correlation of a layer with itself, and that the width of the high correlation band either side of the diagonal is indicative of the vertical distance over which the field is wellcorrelated.

[35] The isochemical cases (Figures 9a-9c) have a fairly broad correlation peak in the lower mantle, with a narrow correlation in the upper mantle (upper right of plot). Filtering broadens the peak, particularly in the upper mantle. 

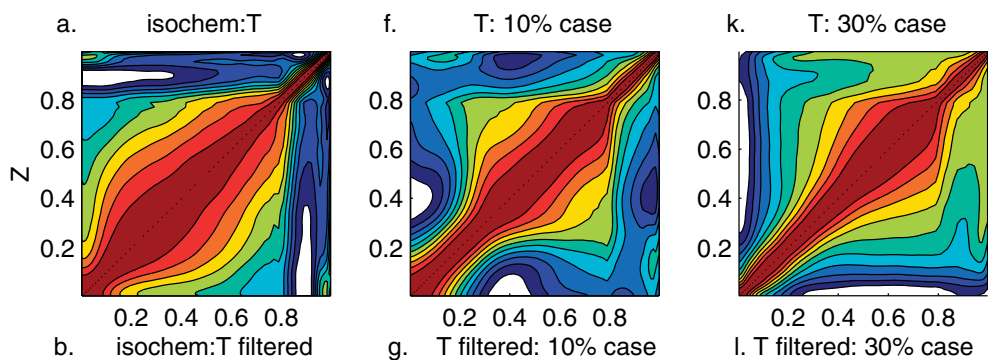

$\begin{array}{llll}0.2 & 0.4 & 0.6 & 0.8\end{array}$

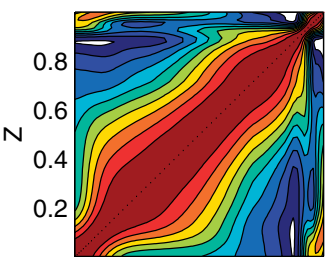

g. T filtered: $10 \%$ case

$\begin{array}{llll}0.2 & 0.4 & 0.6 & 0.8\end{array}$

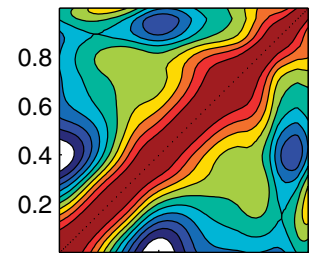

I. T filtered: $30 \%$ case

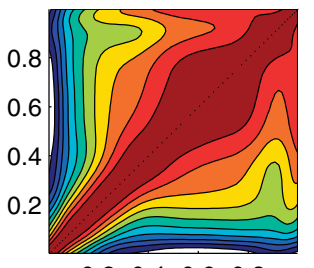

$\begin{array}{llll}0.2 & 0.4 & 0.6 & 0.8\end{array}$
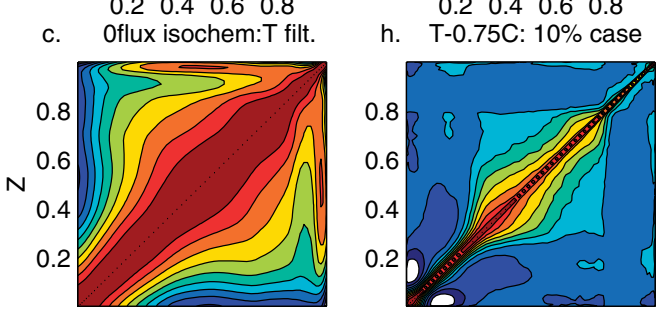

$\begin{array}{llll}0.2 & 0.4 & 0.6 & 0.8\end{array}$
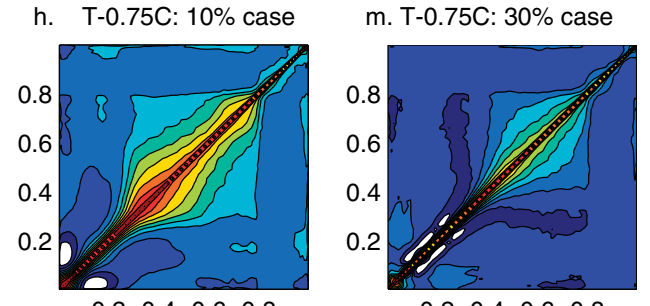

$\begin{array}{llll}0.2 & 0.4 & 0.6 & 0.8\end{array}$

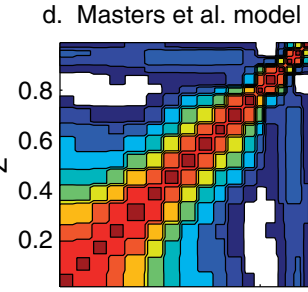

$\begin{array}{llll}0.2 & 0.4 & 0.6 & 0.8\end{array}$

i. T- $0.75 \mathrm{C}$ filt: $10 \%$ case

$\begin{array}{llll}0.2 & 0.4 & 0.6 & 0.8\end{array}$

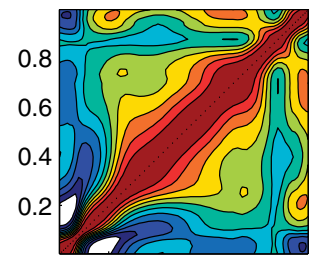

n. T-0.75C filt: $30 \%$ case

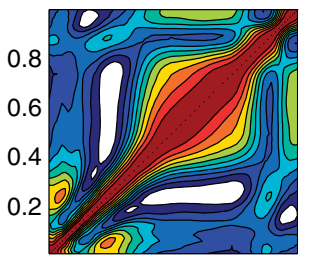

$\begin{array}{llll}0.2 & 0.4 & 0.6 & 0.8\end{array}$

$\begin{array}{llll}0.2 & 0.4 & 0.6 & 0.8\end{array}$

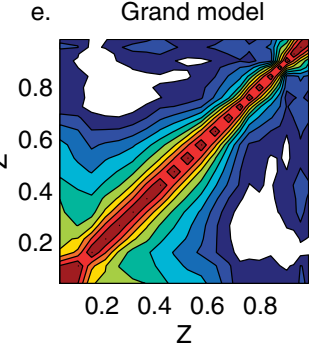

j. Oflux T-0.45C filt: $10 \%$
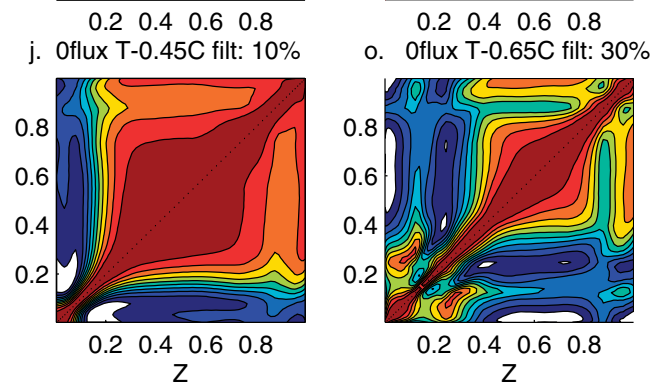

Figure 9. Radial correlation functions for the presented cases. Isochemical: (a) temperature and (b) density for the the fixed CMB case and (c) density for the isochemical zero net flux CMB case. Seismic tomography: (d) seismic tomogram of [Masters et al., 2000]; (e) seismic tomogram of S. P. Grand (updated from [Grand et al., 1997]). Models with 10\% dense material: (f) temperature (g) composition (h) $T-0.75 \mathrm{C}$ and (i) density for the case a fixed $T \mathrm{CMB}$ and (j) density for the case with a zero net flux CMB. Models with 30\% dense material: (k) temperature (l) composition (m) $T-0.75 \mathrm{C}$ and (n) density for the fixed $T \mathrm{CMB}$ case, and (o) density for the model with a zero net flux CMB.

[36] The globally layered case's temperature RCF (Figures 9k and 91) narrows in the deepest part of the mantle (lower left), where the layer exists; the filtered version looks similar. The seismic velocity RCF (Figure $9 \mathrm{~m}$ ) is dramatically different, with a very narrow correlation throughout the mantle. This lack of radial correlation is due to the presence of smallscale chemical heterogeneities in the upper layer caused by entrainment; that is, the $C$ field has very poor radial correlation. When filtered (Figure 9n), the radial correlation becomes broad in the upper half of the lower mantle but distinctively "pinched" 
in the lower half of the lower mantle. The corresponding RCF for the zero net CMB flux case (Figure 9o) is, not surprisingly, very similar.

[37] The temperature RCF for the discontinuously layered, fixed $T$ CMB case (Figures $9 \mathrm{f}-9 \mathrm{~g}$ ) is fairly broad throughout the lower mantle, with filtering having only a small effect. The seismic velocity field (Figure 9h) again has little radial correlation but when filtered (Figure 9i) broadens significantly but with distinctive pinching in the deepest mantle. For the zero net flux CMB version, the RCF is much broader in the lower mantle (Figure 9j).

[38] RCFs for actual seismic tomographic Earth models (Figures 9d and 9e) display a central correlation band that increases slightly toward the base of the mantle, with no hint of the narrowing that would be expected if some type of deep chemical layer were present. However, the strong effect of filtering on the simulated seismic velocity field for the thermochemical cases is disturbing because it implies that the RCF may be dominated by the seismic tomographic process, a complex combination of wave propagation, basis functions, path coverage, relocation, inversion, etc. [Johnson et al., 1993; Megnin et al., 1997] that is only very crudely represented here.

\section{Discussion and Conclusions}

\subsection{Summary of Findings}

\subsubsection{Global layer}

[39] These calculations indicate that a global, deep, dense, undulating layer with strong internal heating produces the following diagnostic features: (1) Enormous volumetric heterogeneity in mid-lower mantle, which is greatly reduced by the partial cancellation of $T$ and $C$ contributions to seismic velocity but unlikely to be completely hidden, exists. The best diagnostic for detecting this heterogeneity is the spectral heterogeneity map. The heterogeneity is associated with (for the $T$ and $C$ fields) the large difference in $T$ or $C$ between the interior of the dense layer and the mantle above, or (for density or seismic velocity fields) the thermochemical boundary layer at the undulating top of the layer, and with the strong linear upwellings that arise from the top of the layer. (2) Very little heterogeneity in the deepest mantle (inside the layer) is present because the low viscosity reduces temperature variations while facilitating vigorous small-scale convection. (3) Strong linear hot upwellings rise from the top of the layer into the upper mantle, even with no net flux across the CMB. (4) A distinctive narrowing of the radial correlation function in the lower $\sim 1000 \mathrm{~km}$ of the mantle is observed, although the RCF is sensitive to the seismic tomographic "filtering." (5) Very high temperatures develop within the dense layer even with no CMB heat flux, which may cause problems with sustaining the geodynamo. Present seismic tomographic models do not display these features, making such a layered model difficult to reconcile with observations. Furthermore, the high deep mantle temperatures would make it difficult to remove heat from the core, leading to problems with Earth's thermal evolution [McNamara and van Keken, 2000].

\subsubsection{Discontinuous piles}

[40] A discontinuous layer, in which dense material is swept into "piles," results in a heterogeneity spectrum somewhat compatible with seismic tomographic models, with strong heterogeneity near the base of the mantle, but much weaker upper boundary heterogeneity than observed. It is likely that the upper boundary heterogeneity would be greatly increased by the inclusion of compositionally buoyant continents [Tackley, 2000c], just as the lower boundary heterogeneity is increased here by the presence of compositionally dense "piles." The radial correlation function for the combined $T$ and $C$ fields ("seismic velocity") displays a narrowing in the deepest mantle that is not observed in seismic tomographic models, although this is much subdued in RCF for the $T$ field alone. Since tomographic models display two large features (often called "superplumes" or "megaplumes") that could be interpreted as thermochemical "piles," due to their size, sharp edges [Ritsema et al., 1998], and positive density anomaly [Ishii and Tromp, 1999] (inconsistent with a purely thermal origin), searching for models in which the $T$ and $C$ con- 
tributions to seismic velocity mostly cancel out, thereby "hiding" the dense material, is probably not the most appropriate paradigm for this scenario, unlike for the continuous layer scenario. The most appropriate "seismic velocity" field for this scenario may thus lie between the $T$ field and $(T-0.75 C)$ field considered here.

\subsubsection{Isochemical convection}

[41] Isochemical convection with these parameters displays a number of interesting features, particularly (1) downwellings that appear to stop some distance above the $\mathrm{CMB}$, although this depends on how the temperature field is plotted and (2) focused hot linear upwellings when internal heating dominates. Both of these have been observed in seismic tomographic models [Su et al., 1992; van der Hilst and Karason, 1999]; the present results thus suggest that it is not necessary to invoke any special mechanism, such as layering, to explain them.

\subsection{Which Model is Most Realistic?}

[42] The presented diagnostics appear to disfavor the existence of a globally continuous deep layer, but there is no clear preference between the "piles" scenario and isochemical convection, since neither of these fit the observations perfectly, though each matches in some respects.

[43] The isochemical case has the most realistic $\mathrm{RCF}$ and a reasonable SHM when a large fraction of CMB heat flow is present (with zero net CMB flux there is too much heterogeneity in the lower mantle compared to top and bottom boundaries). Compositionally buoyant continents would add additonal heterogeneity at the top [Tackley, 2000c].

[44] Realistic heterogeneity is also obtained in the "piles" model, although its RCF has a deep mantle narrowing that is not observed. There are some other possibly unrealistic features of this model. First the strong linear upwellings from the top of the dense material are not observed in seismic tomographic models. However, if when applying this to Earth the "piles" are equated to the two observed superplumes, then the volume of dense material should be significantly lower than in the present numerical model, and the "piles" would be round not linear. These two factors should combine to make the induced upwellings plume-like rather than linear, and indeed, the regions above these superplumes are regions of high hot spot density [Ray and Anderson, 1994], and in the African case, anomalously high tomography [Lithgowbertelloni and Silver, 1998]. A second problem with the "pile" scenario is the negative topography that would be induced under the accumulations of dense material [Davies and Gurnis, 1986], which may be inconsistent with geodetic constraints [Forte et al., 1995]. However, preliminary modeling indicates that with a realistic viscosity contrast, the negative topography can be eliminated or even reversed [Tackley, 2000a], and this is the subject of ongoing study. A third issue is that when the seismically observed superplume under Africa is interpreted as a buoyant hot thermal feature, it is successful in explaining the anomalously high African surface topography [Lithgowbertelloni and Silver, 1998] and its growth [Gurnis et al., 2000]. However, in the presented model, there are strong upwellings that would no doubt cause surface uplift arising from the top of the dense accumulations; furthermore, normal mode splitting data appears to favor the lower part of the superplumes being negatively buoyant [Ishii and Tromp, 1999], which is more consistent with a thermochemical composition. More work needs to be done to reconcile the various observations.

\subsection{Model Shortcomings and Future Directions}

[45] The present model is not completely Earthlike, making it important to discuss what effect the model shortcomings have on the results. The major shortcomings are geometry (Cartesian not spherical), rheology (no temperature-dependence and no plate tectonics), and heating mode (perhaps too much concentration of heat producing elements into the lower layer). Furthermore, two shortcomings of this paper, rather than the model itself, are that only one value of chemical buoyancy ratio $B$ is considered per scenario, and only one chemical initial condition is considered. 


\subsubsection{Rheology and geometry}

[46] The geometric and rheological shortcomings may affect the planform and the nature of upwellings and downwellings. Tectonic plates are generally thought to be dominant in influencing the planform and horizontal length scales of mantle convection [Bunge and Richards, 1996; Davies, 1989; Ratcliff et al., 1997], so their absence may account for the shorter than realistic horizontal length scales in this model. Temperature-dependent viscosity would make the downwellings stronger, suppressing the time-dependent, small-scale structures observed in these results. Strong linear downwellings converging on a CMB that in spherical geometry has a much smaller area than the surface might tend to sweep the dense material into rounded rather than linear accumulations. CMB heat flux and lower mantle temperatures are also influenced by spherical geometry. Regarding upwellings, plume-like upwellings are favored by spherical geometry [Bercovici et al., 1989a, 1989b] and by temperature-dependent viscosity [Tackley, 1993; White, 1988], so upwellings arising from the top of dense material might in reality be plume-like, as they are in laboratory experiments [Davaille, 1999a, 1999b]. In addition, these upwellings would be narrower if temperature-dependent viscosity was included, which may reduce the seismic heterogeneity in the midmantle.

[47] More realistic temperature-dependent viscosity would make the dense, hot material even less viscous, influencing the dynamics. According to best estimates [Yamazaki and Karato, 2001] the total activation enthalpy $\mathrm{H}^{*}$ (i.e., including both the activation energy and activation volume terms) is at least $450 \mathrm{~kJ} / \mathrm{mol}$ for diffusion creep in the lower $\sim 1000 \mathrm{~km}$ of the mantle, so a temperature change from 2500 to $3500 \mathrm{~K}$ would give a viscosity contrast of $\sim 500$, or a change from 2000 to $3000 \mathrm{~K}$ would give a viscosity contrast of 8300 . Of course, this is on top of any intrinsic viscosity contrast due to composition.

\subsubsection{Heating mode}

[48] Internal heating in "statistically steady state" models such as those presented here may represent a combination of radiogenic heating and secular cooling, perhaps in roughly equal proportions. In that case, the present concentration of internal heating into the dense material due to concentration of heat producing elements, is exaggerated. If heating were more evenly distributed, then the dense material would likely become less hot and the upwellings from the top of it would be weaker. This, however, would be offset by an increase in basal heating, particularly for global layering, since the basally injected heat has to pass through the chemical boundary.

\subsubsection{Chemical buoyancy}

[49] What might be the effect of varying the chemical buoyancy parameter $B$ ? The values used here were chosen to maximize topography while still obtaining stable layering; that is, $B$ is as low as possible so that decreasing it much further would lead to instability, overturn, and rapid mixing of the dense material [Olson and Kincaid, 1991]. Alternatively, increasing $B$ would reduce the layer topography. The volumetric lateral heterogeneity that is due to the large difference in $T$ or $C$ between the dense material and regular mantle would thus be confined to a smaller radial extent, and perhaps be less visible overall. The lateral heterogeneity associated with the boundary layer would, however, become focused into a narrower depth range and thus become stronger. In the limiting case with the chemical boundary horizontal, the signature of the thermochemical boundary layer should be at least as strong as that of the CMB boundary layer [Jarvis and Peltier, 1986] and thus obvious.

\subsubsection{Chemical initial condition}

[50] This paper considers only one initial condition: that of a sharp, flat chemical boundary. An important possibility to consider, however, is that the chemical boundary is more gradual, in which case the thermochemical boundary layer that is found here to have a strong signature, may be substantially different. Thermochemical convection in which $C$ initially increased linearly with depth was modeled in 2-D by Hansen and Yuen [2000], who obtained a final outcome resembling the present "piles" case, although they did not look specifically at the sig- 


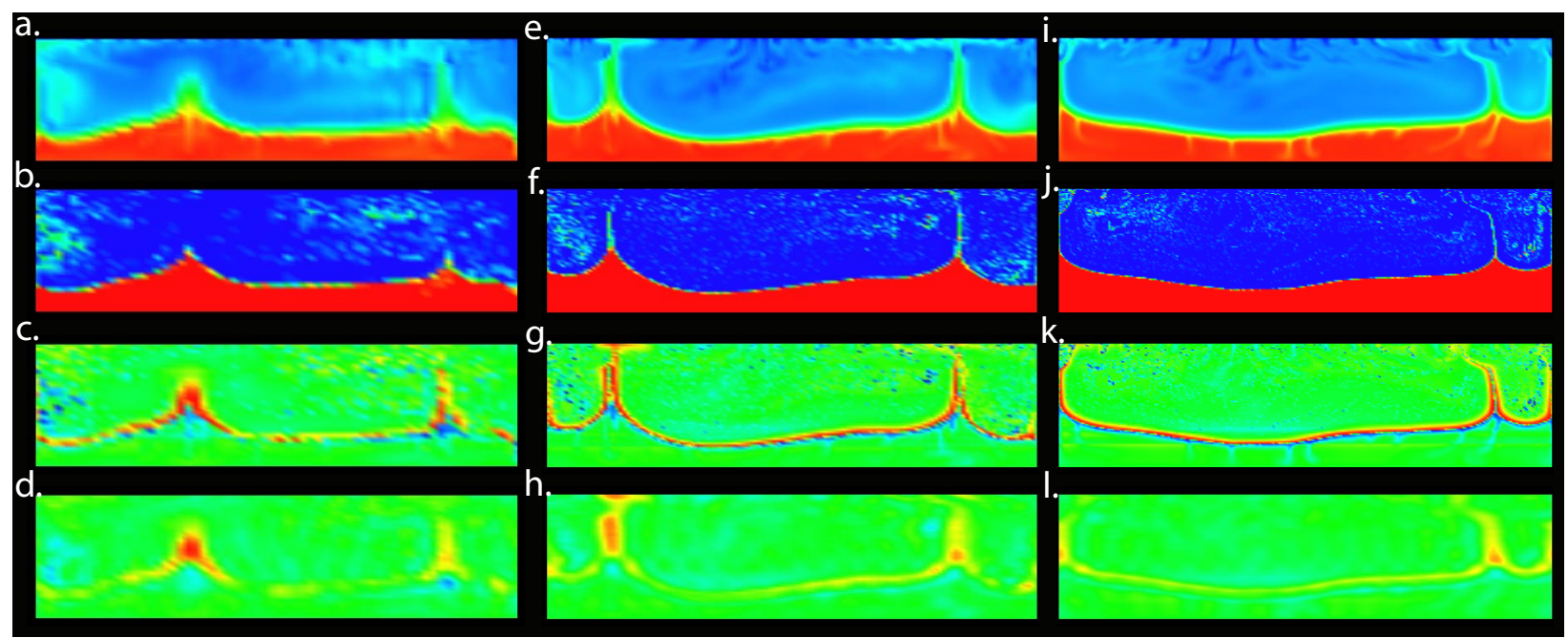

Figure A1. Various two-dimensional fields for the resolution test. (a)-(d) with $64 \times 32$ grid points; (e)-(h) with $128 \times 64$ grid points, and (i) $-(1)$ with $256 \times 96$ grid points. Fields are temperature, scale $0-1$ (Figures A1a, A1e, and A1i); composition, scale 0-1 (Figures A1b, A1f, and A1j); seismic velocity $(T-0.75 \mathrm{C}$ ), scale -0.3 to +0.3 (Figures A1c, A1g, and A1k); and filtered seismic velocity, scale -0.15 to +0.15 (Figures A1d, A1h, and A11).

nature of the thermochemical boundary layer. Physically, a sharp chemical boundary might be the outcome of primordial differentiation of a magma ocean [Solomatov and Stevenson, 1993]. A more gradual boundary might arise if a dense layer grew by gradual accumulation of slab material [Christensen and Hofmann, 1994] or products of reactions between the mantle and core [Kellogg and King, 1993; Knittle and Jeanloz, 1989], although these would best be modeled as continuous processes rather than an initial condition.

\subsubsection{Other possibilities}

[51] It is, of course, possible that Earth is presently in a transient, not steady, thermochemical state, perhaps at the point where a dense layer is becoming unstable and experiencing very high-topography "doming" events [Davaille, 1999a]. This possibility is not addressed by the present modeling. Some other proposed thermochemical structures in the mantle (for a summary see [Tackley, 2000b]) are also not addressed here.

\section{Appendix A: Resolution Test}

[52] In order to determine whether the numerical resolution used in these 3 -D cases $(128 \times 128 \times 64)$ is sufficient for the objectives of this study; cases with higher and lower resolution are presented and compared. In 3-D it is not possible using available resources to increase the resolution much beyond what is used here (mainly because of the number of tracers required) so this test is performed in 2-D. While imperfect, a similar heat flow should give a similar width of convective features compared to the grid spacing in 2-D and 3 -D. Cases with $64 \times 32,128 \times 64$, and $256 \times$ 96 grid cells are presented. The number of tracers is proportional to the number of cells with an average of 12.2 per cell. Cases were run for a nondimensional time of $5 \times 10^{-3}$ ( 2.12 Gyr).

[53] Temperature, composition, and seismic velocity fields (filtered and unfiltered) are compared in Figure A1. Although the overall character of the fields is similar at all resolutions, a major increase in the sharpness of features is observed in going from $64 \times 32$ to $128 \times 64$ cells. The improvement in progressing to $256 \times 96$ cells is only slight in the diffusive temperature field (the difference is less than the time variation of individual solutions) but more noticable in the nondiffusive compositional field. The filtered seismic velocity fields are very similar in character. 

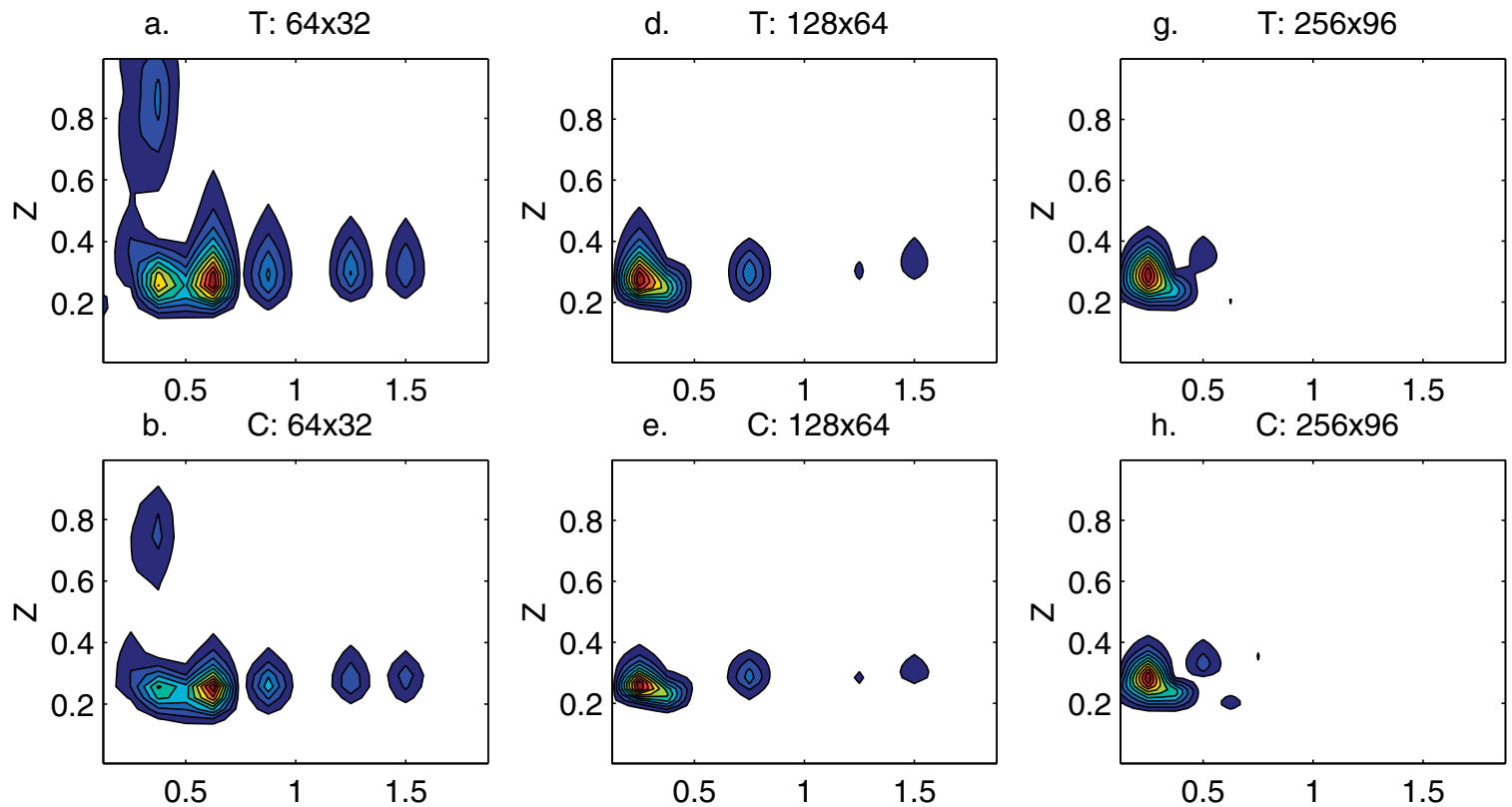

c. density: $64 \times 32$
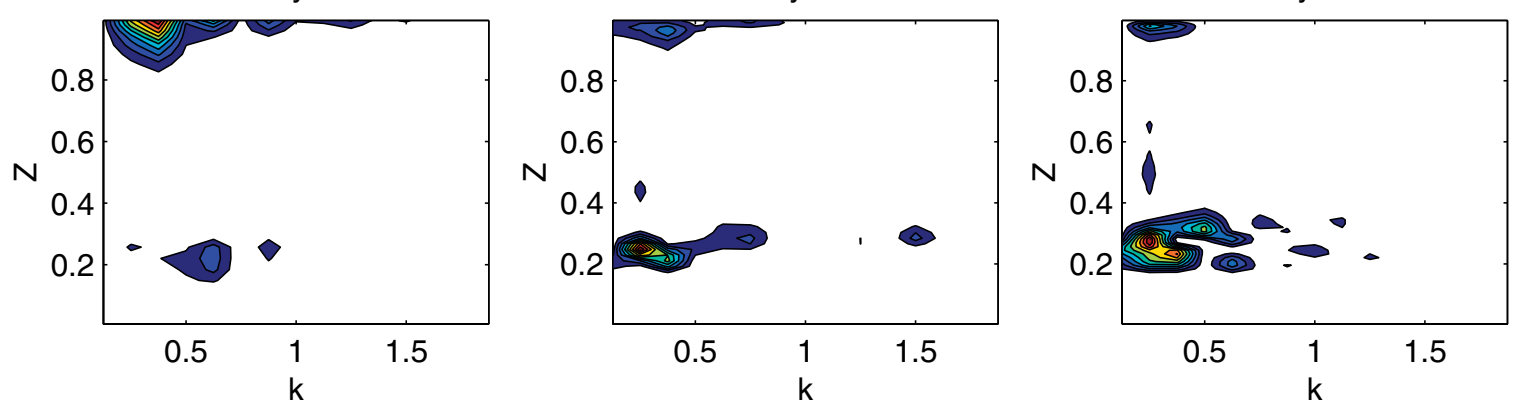

Figure A2. Spectral heterogeneity maps for the two-dimensional resolution test cases. (a) $-(d)$ with $64 \times 32$ grid points; (e)-(h) with $128 \times 64$ grid points, and (i)-(1) with $256 \times 96$ grid points. Fields are temperature (Figures A2a, A2d, and A2g), composition (Figures A2b, A2e, and A2h), density (Figures A2c, A2f, and A2i).

[54] Spectral heterogeneity maps, plotted in Figure A2, show the dominance of middle lower mantle heterogeneity in all cases at all resolutions, demonstrating that this is a robust conclusion. The lowest resolution case has its peak at a higher wave number (shorter wavelength), but this varies in all of the runs due to time-dependence. The field that is most sensitive to variations is the density, since this is related to the difference between the two primary fields. Density SHMs appear to indicate that the middle lower mantle peak gets stronger relative to the upper boundary layer peak as resolution is increased, but again this variation is smaller than the time variation within a single case.

[55] In conclusion, it appears that the main conclusion of this paper, i.e., a thick, deep layer generates very high amplitude middle lower mantle hetero- geneity that is difficult to hide, is robust with respect to resolution. The $64 \times 32$ cells are inadequate to resolve the small-scale details of structures, but $128 \times 64$ cells appear adequate for this purpose.

\section{Acknowledgments}

[56] This research was funded by the David and Lucile Packard Foundation. Constructive reviews by Marc Parmentier, Ulrich Christensen, and Peter van Keken helped improve the manuscript.

\section{References}

Balachandar, S., D. A. Yuen, and D. Reuteler, Time-dependent 3-dimensional compressible convection with depth-dependent properties, Geophys. Res. Lett., 19(22), 2247-2250, 1992.

Balachandar, S., D. A. Yuen, and D. M. Reuteler, Localization of toroidal motion and shear heating in 3-D high Rayleigh 
number convection with temperature-dependent viscosity, Geophys. Res. Lett., 22(4), 477-480, 1995.

Bercovici, D., G. Schubert, and G. A. Glatzmaier, 3-Dimensional Spherical-Models Of Convection In the Earths Mantle, Science, 244(4907), 950-955, 1989a.

Bercovici, D., G. Schubert, and G. A. Glatzmaier, Influence of heating mode on 3-dimensional mantle convection, Geophys. Res. Lett., 16(7), 617-620, 1989b.

Bunge, H. P., and M. A. Richards, The origin of large-scale structure In mantle convection - Effects of plate motions and viscosity stratification, Geophys. Res. Lett., 23(21), 2987-2990, 1996.

Bunge, H. P., M. A. Richards, and J. R. Baumgardner, Effect of depth-dependent viscosity on the planform of mantle convection, Nature, 379(6564), 436-438, 1996.

Christensen, U. R., and A. W. Hofmann, Segregation of subducted oceanic crust In the convecting mantle, J. Geophys. Res., 99(B10), 19,867-19,884, 1994.

Christensen, U. R., and D. A. Yuen, The interaction of a subducting lithospheric slab with a chemical or phase-boundary, J. Geophys. Res., 89(B6), 4389-4402, 1984.

Davaille, A., Simultaneous generation of hotspots and superswells by convection in a heterogeneous planetary mantle, Nature, 402(6763), 756-760, 1999a.

Davaille, A., Two-layer thermal convection in miscible viscous fluids, J. Fluid Mech., 379, 223-253, 1999b.

Davies, G. F., Mantle convection model with a dynamic plate; Topography, heat flow and gravity anomalies, Geophys. J. Int., 98(3), 461-464, 1989.

Davies, G. F., and M. Gurnis, Interaction of mantle dregs with convection; Lateral heterogeneity at the core-mantle boundary, Geophys. Res. Lett., 13(13), 1517-1520, 1986.

Forte, A. M., J. X. Mitrovica, and R. L. Woodward, Seismicgeodynamic determination of the origin of excess ellipticity of the core-mantle boundary, Geophys. Res. Lett., 22(9), 1013-1016, 1995.

Grand, S. P., R. D. van der Hilst, and S. Widiyantoro, Global seismic tomography: A snapshot of convection in the Earth, GSA Today, 7(4), 1-7, 1997.

Gurnis, M., J. X. Mitrovica, J. Ritsema, and H.-J. van Heijst, Constraining mantle density structure using geological evidence of surface uplift rates: The case of the African Superplume, Geochem. Geophys. Geosyst., 1 (Article), 1999GC000035 [26,963 words], 2000. (Available at http:// www.g-cubed.org)

Gurnis, M., and S. Zhong, Generation of long wavelength heterogeneity in the mantle by the dynamic interaction between plates and convection, Geophys. Res. Lett., 18(4), 581-584, 1991.

Hansen, U., and D. A. Yuen, Numerical simulations of thermal-chemical instabilities at the core mantle boundary, Nature, 334(6179), 237-240, 1988.

Hansen, U., and D. A. Yuen, Extended-Boussinesq thermalchemical convection with moving heat sources and variable viscosity, Earth Planet. Sci. Lett., 176(3-4), 401-411, 2000.

Hofmeister, A. M., Mantle values of thermal conductivity and the geotherm from phonon lifetimes, Science, 283(5408), 1699-1706, 1999.
Houseman, G., The dependence of convection planform on mode of heating, Nature, 332(6162), 346-349, 1988.

Ishii, M., and J. Tromp, Normal-mode and free-air gravity constraints on lateral variations in velocity and density of Earth's mantle, Science, 285(5431), 1231-1235, 1999.

Jarvis, G. T., and W. R. Peltier, Lateral heterogeneity in the convecting mantle, J. Geophys. Res., 91(B1), 435-451, 1986.

Johnson, S., G. Masters, P. J. Tackley, and G. A. Glatzmaier, How well can we resolve a convecting Earth with seismic data?, in Eos Trans. AGU, 74(43), Fall Meet. Suppl., F80, 1993.

Jordan, T. H., P. Puster, G. A. Glatzmaier, and P. J. Tackley, Comparisons between seismic Earth structures and mantle flow models based on radial correlation functions, Science, 261(5127), 1427-1431, 1993.

Kellogg, L. H., and S. D. King, Effect of mantle plumes on the growth of $\mathrm{D}^{\prime \prime}$ by reaction between the core and mantle, Geophys. Res. Lett., 20(5), 379-382, 1993.

Kellogg, L. H., B. H. Hager, and R. D. van der Hilst, Compositional stratification in the deep mantle, Science, 283(5409), 1881-1884, 1999.

Knittle, E., and R. Jeanloz, Simulating the core-mantle boundary; An experimental study of high-pressure reactions between silicates and liquid iron, Geophys. Res. Lett., 16(7), 609-612, 1989.

Labrosse, S., J. P. Poirier, and J. L. Le Mouel, On cooling of the Earth's core, Phys. Earth Planet. Interiors, 99(1-2), 117, 1997.

Lithgowbertelloni, C., and P. G. Silver, Dynamic topography, plate driving forces and the african superswell, Nature, 395(6699), 269-272, 1998.

Masters, G., G. Laske, H. Bolton, and A. Dziewonski, The relative behavior of shear velocity, bulk sound speed, and compressional velocity in the mantle: Implications for chemical and thermal structure, in Earth's Deep Interior: Mineral Physics and Tomography From the Atomic to the Global Scale, Geophys. Monogr. Ser., vol. 117, edited by S. Karato, et al., pp. 63-87, AGU, Washington, D.C., 2000.

McNamara, A. K., and P. E. van Keken, Cooling of the Earth: A parameterized convection study of whole versus layered models, Geochem. Geophys. Geosyst., 1, 2001GC000045, 2000. (Available at http://www.g-cubed.org)

Megnin, C., H. P. Bunge, B. Romanowicz, and M. A. Richards, Imaging 3-D spherical convection models - what can seismic tomography tell us about mantle dynamics, Geophys. Res. Lett., 24(11), 1299-1302, 1997.

Montague, N. L., and L. H. Kellogg, Numerical models for a dense layer at the base of the mantle, J. Geophys. Res., 105(B5), 11,101-11,114, 2000.

Moresi, L. N., and V. S. Solomatov, Numerical investigation of $2 \mathrm{~d}$ convection with extremely large viscosity variations, Phys. Fluids, 7(9), 2154-2162, 1995.

Moresi, L., and V. Solomatov, Mantle convection with a brittle lithosphere: Thoughts on the global tectonic styles of the Earth and Venus, Geophys. J. Int., 133(3), 669-682, 1998.

Moresi, L., M. Gurnis, and Z. Shijie, Plate tectonics and convection in the Earth's mantle: toward a numerical simulation, Comput. Sci. Eng., 2(3), 22-33, 2000.

Olson, P., and C. Kincaid, Experiments on the interaction of 
thermal convection and compositional layering at the base of the mantle, J. Geophys. Res., 96(B3), 4347-4354, 1991.

Parmentier, E. M., and C. Sotin, Three-dimensional numerical experiments on thermal convection in a very viscous fluid: Implications for the dynamics of a thermal boundary layer at high Rayleigh number, Phys. Fluids, 12(3), 609-617, 2000.

Parmentier, E. M., C. Sotin, and B. J. Travis, Turbulent 3-D thermal convection in an infinite Prandtl number, volumetrically heated fluid - Implications for mantle dynamics, Geophys. J. Int., 116(2), 241-251, 1994.

Ratcliff, J. T., P. J. Tackley, G. Schubert, and A. Zebib, Transitions in thermal convection with strongly variable viscosity, Phys. Earth Planet. Inter, 102, 201-212, 1997.

Ray, T. W., and D. L. Anderson, Spherical disharmonics in the earth-sciences and the spatial solution - ridges, hotspots, slabs, geochemistry and tomography correlations, J. Geophys. Res., 99(B5), 9605-9614, 1994.

Ritsema, J., S. Ni, D. V. Helmberger, and H. P. Crotwell, Evidence for strong shear velocity reductions and velocity gradients in the lower mantle beneath Africa, Geophys. Res. Lett., 25(23), 4245-4248, 1998.

Schubert, G., Numerical models of mantle convection, Ann. Rev. Fluid Mech., 24, 359-394, 1992.

Schubert, G., and T. Spohn, Two-layer mantle convection and the depletion of radioactive elements in the lower mantle, Geophys. Res. Lett., 8(9), 951-954, 1981.

Solomatov, V. S., Scaling of temperature-dependent and stressdependent viscosity convection, Phys. Fluids, 7(2), 266-274, 1995.

Solomatov, V. S., and D. J. Stevenson, Nonfractional crystallization of a terrestrial magma ocean, J. Geophys. Res., 98(E3), 5391-5406, 1993.

Su, W. J., and A. M. Dziewonski, Simultaneous inversion for 3 -d variations in shear and bulk velocity in the mantle, Phys. Earth Planet. Inter, 100(1-4), 135-156, 1997.

$\mathrm{Su}$, W. J., R. L. Woodward, and A. M. Dziewonski, Deep origin of mid-ocean-ridge seismic velocity anomalies, Nature, 360(6400), 149-152, 1992.

Tackley, P. J., Effects of strongly temperature-dependent viscosity on time-dependent, 3-dimensional models of mantle convection, Geophys. Res. Lett., 20(20), 2187-2190, 1993.

Tackley, P. J., Effects of strongly variable viscosity on threedimensional compressible convection in planetary mantles, J. Geophys. Res., 101(B2), 3311-3332, 1996a.

Tackley, P. J., On the ability of phase transitions and viscosity layering to induce long-wavelength heterogeneity in the mantle, Geophys. Res. Lett., 23(15), 1985-1988, 1996 b.

Tackley, P. J., Three-dimensional simulations of mantle convection with a thermochemical CMB boundary layer: $\mathrm{D}^{\prime \prime}$ ?, in The Core-Mantle Boundary Region, Geodynamic Ser, vol. 28, edited by M. Gurnis, M. E. Wysession, E. Knittle, and B. A. Buffett, pp. 231-253, AGU, Washington, D.C., 1998.

Tackley, P. J., Chemical heterogeneity in the deepest mantle: Dynamical models and observational consequences, EOS Trans. AGU Fall Meet. Suppl., 81(48), F906, 2000a.

Tackley, P. J., Mantle convection and plate tectonics: towards an integrated physical and chemical theory, Science, 288(5473), 2002, 2000b.
Tackley, P. J., The quest for self-consistent incorporation of plate tectonics in mantle convection, in History and Dynamics of Global Plate Motions, Geophys. Monogr. Ser., vol. 121, edited by M. A. Richards, R. Gordon, and R. van der Hilst, AGU, Washington, D.C., 2000c.

Tackley, P. J., Self-consistent generation of tectonic plates in time-dependent, three-dimensional mantle convection simulations, 1, Pseudo-plastic yielding, Geochem. Geophy. Geosyst., 1 (Article), 2000GC000036 [14,503 words], 2000d. (Available at http://www.g-cubed.org)

Tackley, P. J., Self-consistent generation of tectonic plates in time-dependent, three-dimensional mantle convection simulations, 2, Strain weakening and asthenosphere, Geochem. Geophys. Geosyst., 1 (Article), 2000GC000043 [14,420 words] 2000e. (Available at http://www.g-cubed.org)

Tackley, P. J., Convection in Io's asthenosphere: Redistribution of non-uniform tidal heating by mean flows, J. Geophys. Res., 106, 32971, 2001.

Tackley, P. J., D. J. Stevenson, G. A. Glatzmaier, and G. Schubert, Effects of multiple phase transitions in a three-dimensional spherical model of convection in Earth's mantle, $J$. Geophys. Res., 99(B8), 15,877-15,901, 1994.

Tackley, P. J., G. Schubert, G. A. Glatzmaier, P. Schenk, J. T. Ratcliff, and J.-P. Matas, Three-dimensional simulations of mantle convection in Io, Icarus, 149(1), 79-93, 2001.

Travis, B., S. Weinstein, and P. Olson, 3-dimensional convection planforms with internal heat generation, Geophys. Res. Lett., 17(3), 243-246, 1990.

van der Hilst, R. D., and H. Karason, Compositional heterogeneity in the bottom 1000 kilometers of Earth's mantle: Toward a hybrid convection model, Science, 283(5409), 1885-1888, 1999.

van Keken, P. E., and C. J. Ballentine, Dynamical models of mantle volatile evolution and the role of phase transitions and temperature-dependent rheology, J. Geophys. Res., 104(B4), 7137-7151, 1999.

van Keken, P. E., S. Karato, and D. A. Yuen, Rheological control of oceanic crust separation in the transition zone, Geophys. Res. Lett., 23(14), 1821-1824, 1996.

van Keken, P. E., S. D. King, H. Schmeling, U. R. Christensen, D. Neumeister, and M.-P. Doin, A comparison of methods for the modeling of thermochemical convection, J. Geophys. Res., 102(B10), 22,477-22,496, 1997.

Vidale, J. E., G. Schubert, and P. S. Earle, Unsuccessful initial search for a midmantle chemical boundary layer with seismic arrays, Geophys. Res. Lett., 28(5), 859-862, 2001.

Weinstein, S. A., Induced compositional layering in a convecting fluid layer By an endothermic phase-transition, Earth Planet. Sci. Lett., 113(1-2), 23-39, 1992.

White, D. B., The planforms and onset of convection with a temperature-dependent viscosity, J. Fluid Mech., 191, $247-$ 286, 1988.

Yamazaki, D., and S. Karato, Some mineral physics contraints on the rheology and geothermal structure of Earth's lower mantle, Am. Mineral, 86(4), 385-391, 2001.

Zerr, A., A. Diegeler, and R. Boehler, Solidus of Earth's deep mantle, Science, 281(5374), 243-246, 1998. 